TPI-MINN-02/50, UMN-TH-2124/02

ITEP-TH-77/02

\title{
Domain Walls and Flux Tubes in $\mathcal{N}=2$ SQCD: D-Brane Prototypes
}

\author{
M. Shifman ${ }^{a}$ and A. Yung ${ }^{a, b, c}$ \\ ${ }^{a}$ William I. Fine Theoretical Physics Institute, University of Minnesota, \\ Minneapolis, MN 55455, USA \\ ${ }^{b}$ Petersburg Nuclear Physics Institute, Gatchina, St. Petersburg 188300, Russia \\ ${ }^{c}$ Institute of Theoretical and Experimental Physics, Moscow 117250, Russia
}

\begin{abstract}
This paper could have been entitled "D branes and strings from flesh and blood." We study field theoretic prototypes of D branes/strings. To this end we consider $(2+1)$-dimensional domain walls in $(3+1)$-dimensional $\mathcal{N}=2 \mathrm{SQCD}$ with $\mathrm{SU}(2)$ gauge group and $N_{f}=2$ flavors of fundamental hypermultiplets (quarks). This theory is perturbed by a small mass term of the adjoint matter which, in the leading order in the mass parameter, does not break $\mathcal{N}=2$ supersymmetry, and reduces to a (generalized) Fayet-Iliopoulos term in the effective low-energy $\mathcal{N}=2$ SQED. We find 1/2 BPS-saturated domain wall solution interpolating between two quark vacua at weak coupling, and show that this domain wall localizes a U(1) gauge field. To make contact with the brane/string picture we consider the Abrikosov-NielsenOlesen magnetic flux tube in one of two quark vacua and demonstrate that it can end on the domain wall. We find an explicit 1/4 BPS-saturated solution for the wall/flux tube junction. We verify that the end point of the flux tube on the wall plays the role of an electric charge in the dual $2+1$ dimensional SQED living on the wall. Flow to $\mathcal{N}=1$ theory is discussed. Our results lead us to a conjecture regarding the notorious "missing wall" in the solution of Kaplunovsky et al.
\end{abstract}




\section{Introduction}

D branes are extended objects in string theory on which strings can end [1]. Moreover, the gauge fields are the lowest excitations of open superstrings, with the end points attached to D branes. $\mathrm{SU}(N)$ gauge theories are obtained as a field-theoretic reduction of a string theory on the world volume of a stack of $N$ D branes.

In the recent years solitonic objects of the domain wall and string type were extensively studied in supersymmetric gauge theories in $1+3$ dimensions. First, it was observed [2] that there should exist critical (BPS saturated) domain walls in $\mathcal{N}=1$ gluodynamics, with the tension scaling as $N \Lambda^{3}$. (Here $\Lambda$ is the scale parameter.) The peculiar $N$ dependence of the tension prompted [3] a D brane interpretation of such walls. Ideas as to how flux tubes can end on the BPS walls were analyzed [4] at the qualitative level shortly thereafter. Later on, BPS saturated strings and their junctions with domain walls were discussed $[5,6,7]$ in a more quantitative aspect in $\mathcal{N}=2$ theories. Some remarkable parallels between fieldtheoretical critical solitons and the D-brane construction were discovered. In this paper we undertake a systematic investigation of this issue - parallel between fieldtheoretical critical solitons and D branes/strings. The set up which will provide us with multiple tools useful in this endeavor is $\mathcal{N}=2$ SQCD considered by Seiberg and Witten $[8,9]$. Following the original publications, we will introduce a parameter $\mu$ which explicitly breaks $\mathcal{N}=2$ down to $\mathcal{N}=1$. It turns out that in the limit $\mu \ll \Lambda$ we will be able to verify many of the previous conjectures as well as establish new results in the reliable regime of weak coupling.

Research on field-theoretic mechanisms of gauge field localization on the domain walls is an important ingredient of our analysis. The only viable mechanism of gauge field localization was put forward in Ref. [2] where it was noted that if a gauge field is confined in the bulk and is unconfined (or less confined) on the brane, this naturally gives rise to a gauge field on the wall (for further developments see Refs. [10, 11]). Although this idea seems easy to implement, in fact it requires a careful consideration of quantum effects (confinement is certainly such an effect) which is hard to do at strong coupling. This again leads us to models in which the gauge field localization can be implemented at weak coupling. We use, in addition, some new general results [12] (see also Ref. [13]) regarding effective field theories on the critical domain walls in supersymmetric gluodynamics. In the present paper we focus on localization of the Abelian gauge field. The issue of non-Abelian gauge fields on domain walls will be addressed in the subsequent publication.

Our main results can be summarized as follows. First, we suggest an $\mathcal{N}=$ 2 model $(\mathcal{N}=2$ may or may not be softly broken down to $\mathcal{N}=1)$ which possesses both critical walls and strings, at weak coupling. In this model one can address all questions regarding the gauge field localization on the wall and the wall-string junction, and answer these questions in a fully controllable manner. We find that there exists an 1/2 BPS domain wall which does localize a U(1) gauge field; the

charge which presents the source for this field is confined in the bulk. We find that 
an 1/2 BPS flux tube coming from infinity does indeed end on the above wall. The wall-string junction is $1 / 4 \mathrm{BPS}$. When the string ends on the wall the latter is no more flat, it acquires a logarithmic bending which is fully calculable.

In more detail, our theoretical set-up can be described as follows. We consider $(2+1)$-dimensional critical domain walls in $(3+1)$-dimensional SU(2) SQCD originally studied by Seiberg and Witten $[8,9]$. To ensure the existence of domain walls at weak coupling we introduce $N_{f}=2$ flavors of fundamental (quark) hypermultiplets. This theory has a Coulomb branch on which the adjoint scalar acquires an arbitrary vacuum expectation value (VEV),

$$
\langle\Phi\rangle=\langle a\rangle \frac{\tau_{3}}{2},
$$

breaking the $\mathrm{SU}(2)$ gauge group down to $\mathrm{U}(1)$. The Coulomb branch has four singular points in which either monopole, or dyon or one of the two quarks become massless. The first two of these points are always at strong coupling, while the massless quark points can be at weak coupling provided that the quark mass parameters $m_{A}$ are large, $m_{A} \gg \Lambda$, where $A=1,2$ is the flavor index. Below the vacua in which quarks become massless will be referred to as the quark vacua.

In order to have domain walls, the vacuum manifold, rather than being continuous, must consist of isolated points. To guarantee the existence of discrete vacua we perturb the above theory by adding a small mass term for the adjoint matter, via superpotential

$$
\mathcal{W}_{u}=\mu u, \quad u \equiv \operatorname{Tr} \Phi^{2} .
$$

Generally speaking, the superpotential breaks $\mathcal{N}=2$ down to $\mathcal{N}=1$. The Coulomb branch shrinks to four above-mentioned isolated $\mathcal{N}=1$ vacua. Of special importance for what follows is the fact that $\mathcal{N}=2$ supersymmetry is not broken [5, $6,14]$ to the leading order in the parameter $\mu$ in the effective theory. In the effective low-energy SQED the superpotential (1.1) gives rise to a superpotential linear in $a$ plus higher order corrections. If only the linear term in $a$ in the superpotential is kept, the theory is exactly $\mathcal{N}=2$.

We will be mostly interested in the quark vacua since they yield weak coupling regime. Near the quark vacua, to the leading order in $\mathcal{N}=2$ breaking parameter, the superpotential in the effective low-energy SQED is

$$
W_{\mathrm{SQED}}=-\frac{1}{2 \sqrt{2}} \xi a,
$$

where the coefficient $\xi$ is determined by the VEV of the lowest component of $a$ in the given quark vacuum,

$$
\xi=-2 \sqrt{2} \mu\langle a\rangle, \quad\langle a\rangle \sim m_{A} \gg \mu .
$$

The perturbation (1.2) can be "rotated" [6] in such a way as to render it a FayetIliopoulos (FI) term [15]; per se it does not break $\mathcal{N}=2$ supersymmetry. If $\xi \neq 0$, 
the quark fields develop VEV's (of order of $\sqrt{\xi}$ ) breaking $U(1)$ gauge symmetry, so that the theory becomes fully higgsed. Then we consider a domain wall interpolating between the two quark vacua, a task which can be addressed at weak coupling. We also analyze the string-wall junction.

Our domain wall is 1/2 BPS-saturated. It turns out that the solution of the first-order Bogomolny equations can be readily found in the range of parameters

$$
\sqrt{\xi} \ll \Delta m \ll m_{1} \sim m_{2}, \quad \Delta m \equiv m_{1}-m_{2},
$$

when two quark vacua come close to each other on the would-be Coulomb branch. Qualitatively the solution has the following structure: the quark fields are small inside the wall, while $a$ is a slowly varying (almost linear) function of $z$ where $z=x_{3}$ is the coordinate orthogonal to the wall. The original $\mathrm{U}(1)$ gauge field is higgsed outside the wall — this is a "superconducting" phase. Inside the wall superconductivity is destroyed. Correspondingly, magnetic charges are confined in the bulk [5, 16], giving rise to magnetic flux tubes in the bulk ${ }^{1}$, while inside the wall the magnetic flux can spread freely. The U(1) gauge field $A_{m}^{(2+1)}$ localized on the wall, which describes interaction of the probe magnetic charges placed on the wall, is dual to the original $\mathrm{U}(1)$ gauge field.

As well-known [21], 2+1 dimensional gauge field is equivalent to a real scalar (compact) field — we will call it $\sigma$. This must be one of the moduli fields. In the limit of $\mathcal{N}=2$ supersymmetry, the effective field theory on the $1 / 2$ BPS wall must possess four conserved supercharges (i.e. it is $\mathcal{N}=2$ from the $(2+1)$-dimensional standpoint). Then the minimal supersymmetry representation contains two real boson fields - the effective field theory on the wall must include two real boson fields. One is the above mentioned $\sigma(t, x, y)$, another originates from the translational collective coordinate, the position of the wall $z_{0}$. We will refer to this field as $\zeta(t, x, y)$.

In the limit of exact $\mathcal{N}=2$, the field $\sigma(t, x, y)$ is massless, as well as $\zeta(t, x, y)$, and is related to the gauge field strength tensor as follows:

$$
F_{n m}^{(2+1)}=\text { const } \cdot \varepsilon_{n m k} \partial^{k} \sigma,
$$

where $n, m=0,1,2$ and the constant on the right-hand side has dimension of mass. Taking account of higher orders in $\mu$ (i.e. quadratic in $a-\langle a\rangle$ term in the superpotential) breaks $\mathcal{N}=2$ supersymmetry of our macroscopic theory down to $\mathcal{N}=1$. Surprisingly, this does not generate a mass term for the field $\sigma(t, x, y)$, which remains a moduli field. This can be seen in many different ways. One of them is through analyzing the fermion zero modes. A Jackiw-Rebbi type index theorem [22] tells us that fermion zero modes (those unrelated to the supertranslational ones) exist even though $\mathcal{N}=2$ is broken. $\mathcal{N}=1$ supersymmetry requires then a bosonic superpartner, which is the massless field $\sigma(t, x, y)$. Thus, at the level of quadratic in

\footnotetext{
${ }^{1}$ The magnetic flux tube is nothing but the Abrikosov-Nielsen-Olesen (ANO) string [17]. Flux tubes in the Seiberg-Witten theory were studied in [18, 16, 19, 6, 20].
} 
derivative terms, the effective moduli field theory on the domain wall world volume is $\mathcal{N}=2$ (four supercharges). The breaking presumably occurs if higher-derivative terms are taken into account.

Next, completing the theme of the gauge field localization on the wall we proceed to the second aspect of the problem - the issue of how strings originating in the bulk can end on the wall. In string theory, the brane localization of gauge fields is closely related to the possibility for an open string to end on a D brane. Since, as we assert, the BPS walls and flux tubes in $\mathcal{N}=2$ SQCD present a close prototype, it is instructive to study this phenomenon in field theory. That the magnetic field flux tube will end on the wall was already explained above, at a qualitative level. There is no doubt that the phenomenon does take place in our model. Our task is more quantitative, however. We want to find (and do find) a 1/4 BPS solution of the first order Bogomolny equations that describes an ANO flux tube ending on the wall. In other words, attaching a flux tube to $1 / 2$ BPS wall makes the configuration $1 / 4$ BPS. The attachment of the tube gives rise to two effects. First, the wall is now bent, and, second, the field $\sigma(x, y)$ develops a vortex. If $\left\{x_{0}, y_{0}\right\}$ are the coordinates of the tube center on the wall, at large separations $r$ from the center

$$
\sigma(x, y)=\alpha
$$

where $\alpha$ is the polar angle on the two dimensional wall surface (Fig. 1) and $r=$ $\sqrt{\left(x-x_{0}\right)^{2}+\left(y-y_{0}\right)^{2}}$.

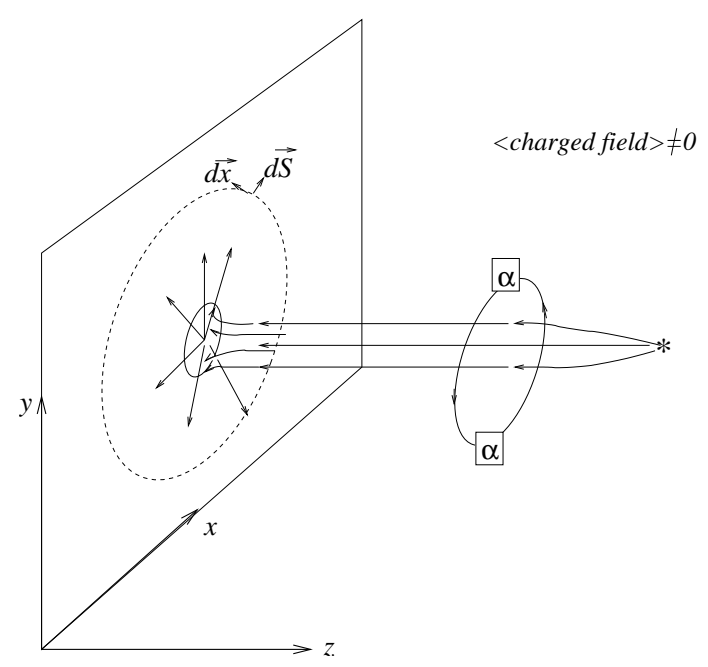

Figure 1: Geometry of the wall-string junction. The gauge field localized on the wall can be cast in the form of a dual $1+2$ dimensional electrodynamics. This field is dual to the gauge field higgsed in the bulk. The probe magnetic charge in the bulk (the magnetic monopole of the original $\mathcal{N}=2$ SQCD) is denoted by asterisk.

According to Eq. (1.5) the vortex in $\sigma$ is equivalent to a radial electric field in 
the dual 1+2 dimensional QED on the wall,

$$
F_{0 i}^{(2+1)}=\mathrm{const} \cdot \frac{x_{i}-\left(x_{0}\right)_{i}}{r^{2}}, \quad i=1,2 .
$$

Thus, the string end point on the wall plays the role of a probe charge for the dual $1+2$ dimensional QED on the wall.

Diminishing the mass parameter $m^{A}$ we move the quark vacua toward the strong coupling regime. (Simultaneously the parameter governing the breaking $\mathcal{N}=2 \rightarrow$ $\mathcal{N}=1$ becomes larger.) At strong coupling there exist two additional vacua, the monopole and dyon ones. A domain wall interpolating between the monopole and dyon vacua was discussed previously [23]. This is a strong coupling problem which requires a "patchy" description — one cannot introduce one and the same effective low-energy theory which would be valid simultaneously near the monopole and dyon vacua because monopoles and dyons are not mutually local states. Nevertheless, we have something new to say regarding the wall interpolating between the monopole and dyon vacua. The analysis of Ref. [23] does not seem to be complete since it does not take into account moduli dynamics on the wall. As is well-established $[24,12,13]$, in $\mathrm{SU}(2)$ theory two distinct critical domain walls (with one and the same tension) must exist. At the same time, only one domain wall was detected in Ref. [23]. We suggest a tentative solution to the paradox of a "missing wall" which should emerge from consideration of the moduli dynamics.

A problem similar to ours was addressed previously in Ref. [7] in the context of (3+1)-dimensional $\mathcal{N}=2$ massive sigma models on hyper-Kähler target spaces (see also Ref. [25]). The results we obtain in $\mathcal{N}=2$ SQCD are in qualitative agreement with those obtained in Ref. [7] in sigma models. In particular in sigma model the existence of a compact moduli field on the wall, representing dual to $\mathrm{U}(1)$ gauge field, was demonstrated, which is certainly no accident. Indeed, in the limit opposite to the that quoted in Eq. (1.4), when $\sqrt{\xi} \gg \Delta m$, the photon and its superpartners become heavy in $\mathcal{N}=2 \mathrm{SQCD}$, and can be integrated out. Then, $\mathcal{N}=2$ SQCD reduces in the low-energy limit to $\mathcal{N}=2$ sigma model with the hyper-Kähler Eguchi-Hanson target space studied in Ref. [7].

The paper is organized as follows. In Sect. 2 we present a toy (nonsupersymmetric) model which has a domain wall and exhibits the phenomenon of the gauge field localization on the wall. Although this model is primitive it serves as a nice illustration for one of the phenomena we are interested in - the occurrence of a $(2+1)$-dimensional gauge field localized on the wall. Section 4 introduces our basic model - supersymmetric QED - obtained as a reduction of the Seiberg-Witten model. We specify in which limit the model has extended $\mathcal{N}=2$ supersymmetry, while for nonlimiting values of parameters it is $\mathcal{N}=1$. In Sect. 5 we derive and solve first order BPS equations for the domain wall interpolating between two quark vacua. Of most importance is Subsec. 5.4 where we derive field theory for the moduli fields living on the wall. In Sect. 6 we review the ANO strings in (the low-energy limit of) the Seiberg-Witten theory. Section 7 treats the issue of strings ending on 
the wall. Here we derive first order BPS equations for the string-wall junctions and discuss the properties of $1 / 4$ BPS solutions to these equations. We find how the magnetic flux which the flux tube brings to the wall spreads out inside the wall. In Sect. 8 we discuss the impact of soft breaking of $\mathcal{N}=2$ down to $\mathcal{N}=1$ in our model. Brief remarks on the literature, including the mystery of a "missing wall" in the solution of Kaplunovsky et al. are presented in Sect. 9.

\section{A toy model}

In this section we will consider a toy (non-supersymmetric) model which exhibits the phenomenon we are interested in - the gauge field localization on a wall. This model lacks certain ingredients which will be of importance in the analysis of more sophisticated supersymmetric models, to be carried out below. The main virtue of the toy model is its simplicity. It will serve as a warm up exercise.

Let us assume that we have two complex fields $\phi$ and $\chi$, with one and the same electric charge, coupled to a U(1) gauge field ("photon"), with the following selfinteraction:

$$
\begin{gathered}
\mathcal{L}_{\text {toy }}=-\frac{1}{4 e^{2}} F_{\mu \nu} F^{\mu \nu}+\left|D_{\mu} \phi\right|^{2}+\left|D_{\mu} \chi\right|^{2}-V(\phi, \chi), \\
V(\phi, \chi)=\frac{\lambda}{2}\left\{\left(\bar{\phi} \phi-v^{2}\right)^{2}+\left(\bar{\chi} \chi-v^{2}\right)^{2}\right\}+\beta|\phi|^{2}|\chi|^{2},
\end{gathered}
$$

where $v, \lambda$ and $\beta$ are positive constants, and we assume, for simplicity, that $\lambda \ll$ $\beta \ll e^{2} \ll 1$. It is easy to see that the model under consideration has two distinct minima (classical vacua):

(i) $\phi$ develops a vacuum expectation value, $\chi$ does not;

(ii) $\chi$ develops a vacuum expectation value, $\phi$ does not.

In the first case $|\phi|=v$, and one can always take $\phi$ to be real and positive (this is nothing but imposing a gauge condition), $\phi=v$. The phase of $\phi$ is eaten up by the vector field, which becomes massive, with mass $m_{V}=\sqrt{2} e v$. The $\chi^{ \pm}$quanta

have mass $m_{\chi}=\sqrt{(\beta-\lambda)} v$. Finally, there is one real field which remains from $\phi$; it can be parametrized as $\phi=v+\eta$ with real $\eta$. The mass term of the $\eta$ field is $2 \lambda v^{2} \eta^{2}$, so that $m_{\phi}=\sqrt{2 \lambda} v$. In the second vacuum the mass of the vector field remains the same while the roles of the $\phi$ and $\chi$ fields interchange, as well as their masses. Note, however, that in neither vacuum there are massless excitations which would make the vacuum manifold continuous. The energies in these two vacua are necessarily degenerate because of the $Z_{2}$ symmetry $\phi \leftrightarrow \chi$ apparent in Eq. (2.1) which is spontaneously broken. Therefore, there must exist a bona fide domain wall interpolating between vacua (i) and (ii).

Although the analytic solution for the domain wall seems to be unknown in the case at hand, it is not difficult to analyze its qualitative features. Let us assume, for 
definiteness, that the wall lies in the $x y$ plane and impose the following boundary condition (to be referred to as "standard"):

$$
\phi \rightarrow v, \quad \chi \rightarrow 0 \text { at } z \rightarrow-\infty ; \quad \chi \rightarrow v, \phi \rightarrow 0 \text { at } z \rightarrow \infty .
$$

Denote the "standard" domain wall solution (centered at $z=0$ ), with the above boundary conditions, as

$$
\phi_{0}(z), \quad \chi_{0}(z)
$$

Then

$$
e^{i \sigma / 2} \phi_{0}\left(z-z_{0}\right), \quad e^{-i \sigma / 2} \chi_{0}\left(z-z_{0}\right)
$$

is obviously a solution too; it represents a family of solutions with the same energy, containing two moduli - the wall center $z_{0}$ and a phase $\sigma$. The occurrence of $z_{0}$ is due to the (spontaneous) breaking of the translational invariance by the given wall solution, while $\sigma$ is due to the (spontaneous) breaking of a global $\mathrm{U}(1)$.

Indeed, the model (2.1) has two U(1) symmetries:

$$
\phi \rightarrow \phi e^{i \gamma}, \quad \chi \rightarrow \chi ; \quad \text { and } \quad \phi \rightarrow \phi, \quad \chi \rightarrow \chi e^{i \beta}
$$

One of these $\mathrm{U}(1)$ — the diagonal combination — is gauged, the other remains global. It is not spontaneously broken in either of the vacua, (i) or (ii). It is broken, however, on the domain wall.

A qualitative sketch of the "standard" domain wall is given in Fig. 2. $\operatorname{Im} \phi=$ $\operatorname{Im} \chi=0$ on the standard solution. The field $\operatorname{Re} \phi$ starts at $v$ at $z \rightarrow-\infty$, while $\operatorname{Re} \chi$ starts at zero. Then $\operatorname{Re} \phi$ decreases as $v\left(1-e^{\sqrt{\lambda} v z}\right)$ and $\operatorname{Re} \chi$ increases as $v e^{\sqrt{\beta} v z}$. There is a crossover at $z=0$, where the roles of $\operatorname{Re} \phi$ and $\operatorname{Re} \chi$ interchange: the field which was lighter becomes heavier, and vice versa. If one's goal is calculation of the wall tension, one may treat the moduli $z_{0}$ and $\sigma$ as constants. Then the vector field $A_{\mu}$ is not excited, $A_{\mu}=0$. The wall has a two-component structure: the thickness of one component of the wall is $1 /(\sqrt{\lambda} v)$ while that of the other $1 /(\sqrt{\beta} v)$. The latter size is much smaller than the former provided that $\beta \gg \lambda$, as was assumed. The wall tension $T_{\mathrm{w}}$ is saturated by the contribution of the second (narrow) component,

$$
T_{\mathrm{w}} \sim v^{3} \sqrt{\beta}
$$

Our task is more than just calculating the wall tension. We want to construct an effective $2+1$ dimensional field theory for the moduli on the wall world volume. As we will see momentarily, to this end we will have to take into account the third component of the wall, built of the gauge field, which has thickness of order of $1 /(e v)$ (see the zigzagy line in Fig. 2). This component does not show up in the calculation of $T_{\mathrm{w}}$.

Upon quantization, the moduli $z_{0}$ and $\sigma$ become fields (adiabatically) depending on $x_{m} \equiv\{t, x, y\}$. We will call them $\zeta(t, x, y)$ and $\sigma(t, x, y)$, respectively. Furthermore, in Eq. (2.4) the collective coordinates $z_{0}$ and $\sigma$ are to be replaced by

$$
z_{0} \longrightarrow \zeta(t, x, y), \quad \sigma \longrightarrow \sigma(t, x, y) \text {. }
$$




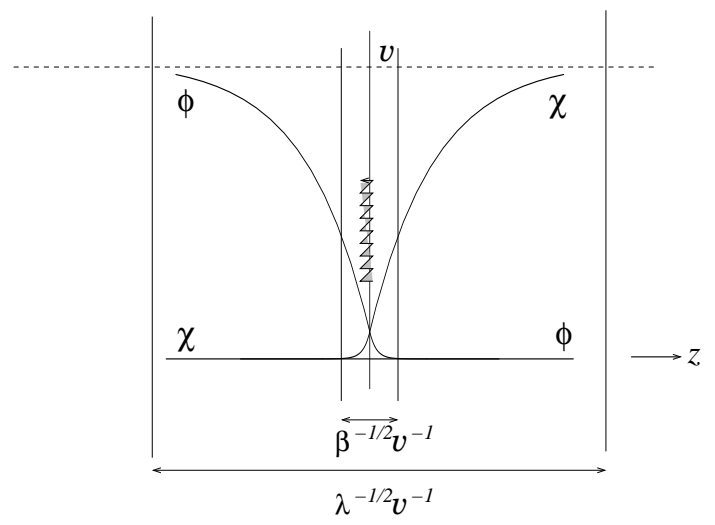

Figure 2: A schematic rendition of the domain wall in the model (2.1).

Then we substitute the expressions for $\phi$ and $\chi$ in the Lagrangian (2.1), integrate over $z$ and obtain in this way a low-energy Lagrangian for the moduli. For $\zeta(t, x, y)$ this procedure is quite standard, leading to

$$
\mathcal{L}_{\zeta}=\frac{T_{\mathrm{w}}}{2}\left(\partial_{m} \zeta\right)\left(\partial^{m} \zeta\right), \quad m=0,1,2 .
$$

For $\sigma(t, x, y)$ there is a subtlety. Indeed, substituting Eq. (2.4) in Eq. (2.1), we arrive at

$$
\bar{\phi}_{0} \phi_{0}\left|\frac{1}{2} \partial_{m} \sigma(t, x, y)-A_{m}\right|^{2}+\bar{\chi}_{0} \chi_{0}\left|\frac{1}{2} \partial_{m} \sigma(t, x, y)+A_{m}\right|^{2} .
$$

It is clear that at $z \rightarrow \pm \infty$ one must choose $A_{m}$ as follows:

$$
A_{m}(t, x, y, z) \rightarrow\left\{\begin{array}{l}
\frac{1}{2} \partial_{m} \sigma(t, x, y) \text { at } z \rightarrow-\infty, \\
-\frac{1}{2} \partial_{m} \sigma(t, x, y) \text { at } z \rightarrow \infty,
\end{array}\right.
$$

i.e. $A_{m}$ is pure gauge. However, at $|z| \lesssim(e v)^{-1}$ the field $A_{m}(t, x, y, z)$ must smoothly interpolate between two regimes in Eq. (2.10) and, hence, cannot be pure gauge in this domain of $z$ (see Fig. 2). It must be chosen in such a way as to minimize the coefficient of $\left(\partial_{m} \sigma\right)^{2}$ in $\mathcal{L}_{\sigma}$. Thus, at $|z| \lesssim(e v)^{-1}$ the photon field strength tensor is generated, with necessity,

$$
F_{m 3} \sim \frac{\partial_{m} \sigma}{\Delta z}, \quad \Delta z=(e v)^{-1} .
$$

Therefore,

$$
\mathcal{L}_{\sigma}=\kappa \frac{v}{e}\left(\partial_{m} \sigma\right)\left(\partial^{m} \sigma\right),
$$

where $\kappa$ is a numerical coefficient of order 1 depending on the form of $A_{m}$ in the intermediate domain. It is determined through minimization. Dynamics of the moduli fields on the wall world sheet is thus described by the Lagrangian

$$
\mathcal{L}_{2+1}=\kappa \frac{v}{e}\left(\partial_{m} \sigma\right)\left(\partial^{m} \sigma\right)+\frac{T_{\mathrm{w}}}{2}\left(\partial_{m} \zeta\right)\left(\partial^{m} \zeta\right) .
$$


The target space of the field $\sigma$ is $S_{1}$,

$$
0 \leq \sigma \leq 2 \pi
$$

As was noted by Polyakov [21], in $2+1$ dimensions, gauge field is equivalent to a compact scalar field, through the relation

$$
F_{m n}^{(2+1)}=\frac{e_{2+1}^{2}}{2 \pi} \varepsilon_{m n k} \partial^{k} \sigma
$$

where $e_{2+1}^{2}$ is $2+1$ dimensional gauge coupling (in our case $e_{2+1}^{2} /\left(8 \pi^{2}\right)=\kappa v / e$ ). As a result, the moduli Lagrangian (2.13) can be rewritten as

$$
\mathcal{L}_{2+1}=-\frac{1}{4 e_{2+1}^{2}} F_{m n} F^{m n}+\frac{T_{\mathrm{w}}}{2}\left(\partial_{m} \zeta\right)\left(\partial^{m} \zeta\right)
$$

The consideration above bears purely illustrative character, first and foremost because it was purely classical. The impact of quantum corrections will be discussed in supersymmetric setting.

\section{Gauge invariant definition of the phase collec- tive coordinate $\sigma$}

When we speak of the relative phase of the fields $\phi$ and $\chi$ we compare their phases in distant points, $z \rightarrow \pm \infty$. In the theory with the local gauge invariance this raises the question as to the meaningfulness of this comparison. The way we have introduced the phase collective coordinate $\sigma$ in Sect. 2 is meaningful only in a specific gauge. In fact, it is useful to give a gauge invariant definition. Let us introduce $\sigma$ as follows:

$$
\sigma=\operatorname{Arg} \phi\left(z_{1}\right)-\operatorname{Arg} \chi\left(z_{2}\right)+\int_{z_{1}}^{z_{2}} d z A_{3}(z)
$$

where formally $z_{1} \rightarrow-\infty, z_{2} \rightarrow \infty$. Under the gauge transformations

$$
\phi(z) \rightarrow e^{i \alpha(z)} \phi(z), \quad \chi(z) \rightarrow e^{i \alpha(z)} \chi(z), \quad A_{3}(z) \rightarrow A_{3}(z)+\partial_{z} \alpha(z),
$$

while $\sigma$ as defined in Eq. (3.1) stays intact.

Although formally $z_{1} \rightarrow-\infty, z_{2} \rightarrow \infty$, there is an unquestionable tradition to set $\phi, \quad \chi=$ const, $A_{\mu}=0$ in the plane vacuum (this is the unitary gauge). Therefore, practically one can take $z_{1}$ just to the left of the wall while $z_{2}$ just to the right. With this choice of $z_{1,2}$ it is perfectly clear that $\sigma$ is a collective coordinate characterizing the wall structure, an internal phase.

Alternatively one can consider a (nonlocal gauge invariant) order parameter

$$
\left\langle\bar{\chi}\left(z_{2}\right) e^{i \int_{z_{1}}^{z_{2}} d z A_{3}(z)} \phi\left(z_{1}\right)\right\rangle .
$$


It obviously vanishes in the plane vacuum, while it reduces to $v^{2} e^{i \sigma}$ if there is a domain wall of the type discussed in Sect. 2. This order parameter is noninvariant under the "second" U(1) — the one which is not gauged. The fact that (3.3) does not vanish on the wall means that the global $\mathrm{U}(1)$ is spontaneously broken triggering the emergence of the Goldstone boson localized on the wall and described by the field $\sigma(t, x, y)$.

\section{Basic model}

\subsection{The original non-Abelian theory}

The field content of $\mathcal{N}=2 \mathrm{SQCD}$ with the gauge group $\mathrm{SU}(2)$ and $N_{f}$ flavors of the quark multiplets is well-known. The $\mathcal{N}=2$ vector multiplet consists of the gauge field $A_{\mu}^{a}$, two Weyl fermions $\lambda_{\alpha}^{f a}$ and the scalar field $\phi^{a}$, all in the adjoint representation of the gauge group. Here $a=1,2,3$ is the color index, $\alpha=1,2$ is the spinor index and $f=1,2$ is an $\mathrm{SU}(2)_{R}$ index (warning: $\mathrm{SU}(2)_{R}$ is not to be confused with the gauge $\mathrm{SU}(2)$ or flavor $\mathrm{SU}(2)$ emerging at $N_{f}=2$, the case to be considered below).

On the Coulomb branch, the adjoint scalar develops a VEV,

$$
\left\langle\phi^{3}\right\rangle \equiv\langle a\rangle
$$

breaking the gauge group down to $\mathrm{U}(1)$. The $\mathrm{W}$ bosons and their superpartners become heavy (with masses of order of $\langle a\rangle \sim m_{A} \gg \Lambda$ ) and can be integrated out. What is left of the vector multiplet in the low-energy SQED are the third color components of $A, \lambda$ and $\phi$, to be referred as $A_{\mu}$, two Weyl fermions $\lambda_{\alpha}^{f}$, and the scalar field $a$. This is the field content of SQED.

The quark multiplets of the non-Abelian theory consist of complex scalar fields $q^{k A}$ and $\tilde{q}_{A k}$ and Weyl fermions $\psi^{k A}$ and $\tilde{\psi}_{A k}$, all in the fundamental representation of the gauge group. Here $k=1,2$ is the color index while $A$ is the flavor index. In what follows we will limit ourselves to $A=1,2$.

With the gauge symmetry spontaneously broken by the condensate (4.1), only the upper $(k=1)$ components, or only the lower $(k=2)$ components of the fields $q, \tilde{q}, \psi$ and $\tilde{\psi}$ remain light in the quark vacua, while the opposite components acquire the same mass as the $\mathrm{W}$ bosons and the other fields in the $\mathcal{N}=2$ vector supermultiplet. They can be integrated out and will play no role in our consideration.

Let us denote the light quark fields as $q^{A}, \tilde{q}_{A}$ and $\psi^{A}$, and $\tilde{\psi}_{A}$, respectively. Note that the scalars form a doublet under the action of global $\mathrm{SU}(2)_{R}$ group, $q^{f}=(q, \overline{\tilde{q}})$. In terms of these fields the bosonic part of the low-energy effective SQED takes the 
form $^{2}$

$$
\begin{aligned}
S_{\mathrm{low}-\mathrm{en}} & =\int d^{4} x\left\{\frac{1}{4 g^{2}} F_{\mu \nu}^{2}+\frac{1}{g^{2}}\left|\partial_{\mu} a\right|^{2}+\bar{\nabla}_{\mu} \bar{q}_{A} \nabla_{\mu} q^{A}+\bar{\nabla}_{\mu} \tilde{q}_{A} \nabla_{\mu} \overline{\tilde{q}}^{A}\right. \\
& +\frac{g^{2}}{8}\left(\left|q^{A}\right|^{2}-\left|\tilde{q}_{A}\right|^{2}\right)^{2}+\frac{g^{2}}{2}\left|\tilde{q}_{A} q^{A}-\frac{f(a)}{2}\right|^{2} \\
& \left.+\frac{1}{2}\left(\left|q^{A}\right|^{2}+\left|\tilde{q}_{A}\right|^{2}\right)\left|a+\sqrt{2} m_{A}\right|^{2}\right\}
\end{aligned}
$$

where $\nabla_{\mu}=\partial_{\mu}-\frac{i}{2} A_{\mu}, \bar{\nabla}_{\mu}=\partial_{\mu}+\frac{i}{2} A_{\mu}$, while near the quark vacua ${ }^{3}$

$$
f(a)=\xi-2 \sqrt{2} \mu \delta a+\ldots
$$

and the ellipses denote terms quadratic in $\delta a$. Under our choice of parameters the generalized FI parameter $\xi$ can be chosen as

$$
\xi=4 \mu m
$$

where

$$
m=\frac{1}{2}\left(m_{1}+m_{2}\right) .
$$

In order to keep $\xi$ real (as we will always do) we assume that both $\mu$ and $m$ are real. The scalar potential in Eq. (4.2) comes from $F$ and $D$ terms of the vector and matter supermultiplets.

\section{$4.2 \mathcal{N}=2$ SQED}

In what follows we will mostly ignore the $a$ dependence of the function $f(a)$ in the second line of Eq. (4.2) setting $f(a)=\xi$. The theory we get in this limit is $\mathcal{N}=2$ SQED. The relative impact of the terms linear in $\delta a$ in the function $f(a)$ is of order $m^{-1} \delta a$. As will be seen momentarily, $\delta a \sim m_{2}-m_{1}$. Therefore, taking account of the dependence of $f(a)$ on $a$ results in a small $(\mathcal{N}=2$ breaking) correction provided $|\Delta m| \ll m$, as we always assume. All our conclusions remain unchanged.

From Eq. (4.2) we can immediately infer the vacuum structure of the model at hand. We have two quark vacua, the first one located at

$$
a=-\sqrt{2} m_{1}
$$

\footnotetext{
${ }^{2}$ Here and below we use a formally Euclidean notation, e.g. $F_{\mu \nu}^{2}=2 F_{0 i}^{2}+F_{i j}^{2},\left(\partial_{\mu} a\right)^{2}=\left(\partial_{0} a\right)^{2}+$ $\left(\partial_{i} a\right)^{2}$, etc. This is appropriate as long as we after static (time-independent) field configurations, and $A_{0}=0$. Then the Euclidean action is nothing but the energy functional. Furthermore, we define $\sigma^{\alpha \dot{\alpha}}=(1,-i \vec{\tau}), \bar{\sigma}_{\dot{\alpha} \alpha}=(1, i \vec{\tau})$. Lowing and raising of spinor indices is performed by virtue of the antisymmetric tensor defined as $\varepsilon_{12}=\varepsilon_{\dot{1} \dot{2}}=1, \varepsilon^{12}=\varepsilon^{i \dot{2}}=-1$.

${ }^{3}$ In our case the variable $u$ defined in Eq. (1.1) can be represented as $u=a^{2} / 2=(1 / 2)(-\sqrt{2} m+$ $\delta a)^{2}$. Hence $f(a)=-2 \sqrt{2} \mu \partial u / \partial a=4 \mu m-2 \sqrt{2} \mu \delta a$.
} 
while the second one at

$$
a=-\sqrt{2} m_{2} .
$$

In the first vacuum the first quark flavor develops a VEV,

$$
q^{1}=\tilde{q}_{1}=\sqrt{\frac{\xi}{2}}, \quad q^{2}=\tilde{q}_{2}=0,
$$

completely breaking the $\mathrm{U}(1)$ gauge symmetry. In the second vacuum the second flavor develops a VEV,

$$
q^{2}=\tilde{q}_{2}=\sqrt{\frac{\xi}{2}}, \quad q^{1}=\tilde{q}_{1}=0,
$$

which does the same job.

Above, the vacuum expectation values of the squark fields were assumed to be real. In fact, one can assign arbitrary phases to the two squark fields. We will discuss the impact of these phases shortly.

Now let us discuss the mass spectrum of light fields in both quark vacua. Consider for definiteness the first vacuum, Eq. (4.6). The spectrum can be obtained by diagonalizing the quadratic form in (4.2). This is done in Ref. [6]; the result is as follows: one real component of field $q^{1}$ is eaten up by the Higgs mechanism to become the third components of the massive photon. Three components of the massive photon, one remaining component of $q^{1}$ and four real components of the fields $\tilde{q}_{1}$ and $a$ form one long $\mathcal{N}=2$ multiplet ( 8 boson states +8 fermion states), with mass

$$
m_{\gamma}^{2}=\frac{1}{2} g^{2} \xi .
$$

The second flavor $q^{2}, \tilde{q}_{2}$ (which does not condense in this vacuum) forms one short $\mathcal{N}=2$ multiplet ( 4 boson states +4 fermion states), with mass $\Delta m$ which is heavier than the mass of the vector supermultiplet ${ }^{4}$. The latter assertion applies to the regime (1.4). In this regime the $W$-boson supermultiplet is heavier still.

If we consider the limit opposite to that in Eq. (1.4) and tend $\Delta m \rightarrow 0$, the "photonic" supermultiplet becomes heavier than that of $q^{2}$, the second flavor field. therefore, it can be integrated out, leaving us with the theory of massless moduli from $q^{2}$, which interact through a nonlinear sigma model with the Kähler term corresponding to the Eguchi-Hanson metric.

In the second vacuum the mass spectrum is similar - the roles of the first and the second flavors are interchanged.

Our immediate goal is constructing $1 / 2$ BPS domain wall interpolating between the above two vacua.

\footnotetext{
${ }^{4}$ In estimating the relative masses of various fields we will ignore the factors of $g^{2}$. Although the coupling constant is certainly assumed to be small to make the theory weakly coupled, we consider the $\mu, m$ dependences as more important.
} 


\section{The domain wall in $\mathcal{N}=2$ SQED}

In this section we work out and solve the first order Bogomolny equations for the domain wall. The Bogomolny equations can be derived in two ways: by performing the Bogomolny completion [26] and by analyzing the set of supercharges and isolating those four that annihilate the wall [27, 28, 2, 29, 30, 31]. We will follow both routes.

\subsection{First-order equations}

First, let us note that the structure of the vacuum condensates in both vacua (4.8) and (4.9) suggests that we can look for domain wall solution using the ansatz

$$
q^{A}=\overline{\tilde{q}}_{A} \equiv \frac{1}{\sqrt{2}} \varphi^{A}
$$

where we introduce a new complex field $\varphi^{A}$. In this ansatz SQED under consideration reduces to

$$
\begin{aligned}
S_{\mathrm{red} S Q E D} & =\int d^{4} x\left\{\frac{1}{4 g^{2}} F_{\mu \nu}^{2}+\frac{1}{g^{2}}\left|\partial_{\mu} a\right|^{2}+\bar{\nabla}_{\mu} \bar{\varphi}_{A} \nabla_{\mu} \varphi^{A}\right. \\
& \left.+\frac{g^{2}}{8}\left(\left|\varphi^{A}\right|^{2}-\xi\right)^{2}+\frac{1}{2}\left|\varphi^{A}\right|^{2}\left|a+\sqrt{2} m_{A}\right|^{2}\right\} .
\end{aligned}
$$

This Lagrangian is qualitatively very similar to that considered in Sec. 2. In particular, it has two global U(1) symmetries, allowing one to independently rotate the fields of the first and second flavors, respectively. The diagonal U(1) is gauged.

In terms of the fields $\varphi^{A}$ the quark condensate becomes

$$
\varphi^{1}=\sqrt{\xi} \exp (i \alpha), \quad \varphi^{2}=0
$$

in the vacuum (4.6), and

$$
\varphi^{2}=\sqrt{\xi} \exp \left(i \alpha^{\prime}\right), \quad \varphi^{1}=0
$$

in the vacuum (4.7). Because of the gauge freedom, the phases $\alpha, \alpha^{\prime}$ can be always chosen to vanish.

If we assume that all fields depend only on the coordinate $z=x_{3}$ the Bogomolny completion of the wall energy functional can be written as

$$
\begin{aligned}
T_{\mathrm{w}} & =\int d z\left\{\left|\nabla_{z} \varphi^{A} \pm \frac{1}{\sqrt{2}} \varphi^{A}\left(a+\sqrt{2} m_{A}\right)\right|^{2}\right. \\
& \left.+\left|\frac{1}{g} \partial_{z} a \pm \frac{g}{2 \sqrt{2}}\left(\left|\varphi^{A}\right|^{2}-\xi\right)\right|^{2} \pm \frac{1}{\sqrt{2}} \xi \partial_{z} a\right\} .
\end{aligned}
$$


Here we do not assume a priori that the gauge field vanishes. We will see that although the gauge field strength does vanish on the flat wall solution at rest, the gauge potential need not vanish. Putting mod-squared terms to zero gives us the first order Bogomolny equations, while the surface term (the last one in Eq. (5.5)) gives the wall tension. Assuming for definiteness that $\Delta m>0$ and choosing the upper sign in (5.5) we get the BPS equations,

$$
\begin{aligned}
\nabla_{z} \varphi^{A} & =-\frac{1}{\sqrt{2}} \varphi^{A}\left(a+\sqrt{2} m_{A}\right), \\
\partial_{z} a & =-\frac{g^{2}}{2 \sqrt{2}}\left(\left|\varphi^{A}\right|^{2}-\xi\right) .
\end{aligned}
$$

These first order equations should be supplemented by the following boundary conditions

$$
\begin{aligned}
\varphi^{1}(-\infty) & =\sqrt{\xi}, \quad \varphi^{2}(-\infty)=0, \quad a(-\infty)=-\sqrt{2} m_{1} ; \\
\varphi^{1}(\infty) & =0, \quad \varphi^{2}(\infty)=\sqrt{\xi}, \quad a(\infty)=-\sqrt{2} m_{2},
\end{aligned}
$$

which show that our wall interpolates between the two quark vacua. As was mentioned, this boundary condition is not generic (it was referred to as "standard" in Sec. 2). The existence of the exact ungauged U(1) implies that the generic boundary condition could be obtained from Eq. (5.7) by multiplying $\phi^{1}$ in the first line by $e^{i \sigma / 2}$ and $\phi^{2}$ in the second line by $e^{-i \sigma / 2}$.

Finally, the tension of the wall satisfying the above equations is

$$
T_{\mathrm{w}}=|(\Delta m) \xi|
$$

Now, let us derive the Bogomolny equations by analyzing relevant combinations of supercharges. We will show that four combinations of supercharges act trivially. To see this explicitly, let us write down the supersymmetry transformations in SQED:

$$
\begin{aligned}
& \delta \lambda^{f \alpha}=\frac{1}{2}\left(\sigma_{\mu} \bar{\sigma}_{\nu} \varepsilon^{f}\right)^{\alpha} F_{\mu \nu}+\varepsilon^{\alpha p} D^{a}\left(\tau^{a}\right)_{p}^{f}+i \sqrt{2} \not^{\alpha \dot{\alpha}} a \bar{\varepsilon}_{\dot{\alpha}}^{f} \\
& \delta \psi^{\alpha A}=i \sqrt{2} \nabla^{\alpha \dot{\alpha}} q^{f{ }_{\bar{\varepsilon}}} \bar{\varepsilon}_{f \dot{\alpha}}+\sqrt{2} \varepsilon^{\alpha f} F_{f}^{A} \\
& \delta \tilde{\psi}_{A}^{\alpha}=i \sqrt{2} \nabla^{\alpha \dot{\alpha}} \bar{q}_{A}^{f} \bar{\varepsilon}_{f \dot{\alpha}}+\sqrt{2} \varepsilon^{\alpha f} \bar{F}_{f A}
\end{aligned}
$$

where we explicitly write out the $\mathrm{SU}(2)_{R}$ indices $f, p=1,2$. Here $D^{a}$ is the $\mathrm{SU}(2)_{R}$ triplet of $D$ terms which in the ansatz (5.1) reduce to

$$
D^{1}=i \frac{g^{2}}{2}\left(\left|\varphi^{A}\right|^{2}-\xi\right), D^{2}=D^{3}=0
$$


while $F^{f}$ and $\bar{F}_{f}$ are the matter $F$ terms,

$$
F^{f A}=i \frac{1}{\sqrt{2}}\left(a+\sqrt{2} m_{A}\right) q^{f A}, \quad \bar{F}_{A f}=i \frac{1}{\sqrt{2}}\left(\bar{a}+\sqrt{2} m_{A}\right) \bar{q}_{A f} .
$$

The fact that the wall we work with is critical implies that some of $\delta \lambda$ and $\delta \psi$ in Eq. (5.9) vanish. Accepting, as above, the ansatz (5.1) and taking into account that all fields depend only on $z$ we get the same first order equations (5.6), provided that $\varepsilon^{\alpha f}$ and $\bar{\varepsilon}_{\dot{\alpha}}^{f}$ satisfy the following conditions

$$
\begin{array}{ll}
\bar{\varepsilon}_{\dot{2}}^{2}=-i \varepsilon^{21}, & \bar{\varepsilon}_{\dot{2}}^{1}=-i \varepsilon^{22}, \\
\bar{\varepsilon}_{\dot{1}}^{1}=i \varepsilon^{12}, & \bar{\varepsilon}_{\dot{1}}^{2}=i \varepsilon^{11} .
\end{array}
$$

These four constraints on the supertransformation parameters show which particular linear combinations of the supercharges act trivially on the domain wall solution. With these four constraints we reduce the number of trivially acting supercharges to four (out of eight). Thus, our domain wall is $1 / 2$ BPS-saturated.

\subsection{Finding the domain wall solution}

Now let us work out the solution to the first order equations (5.6), assuming the conditions (1.4) to be satisfied. The range of variation of the field $a$ inside the wall is of the order of $\Delta m$ (see Eq. (5.7)). The minimization of its kinetic energy implies this field to be slowly varying. Therefore, we may safely assume that the wall is thick; its size $R \gg 1 / \sqrt{\xi}$. This fact will be confirmed shortly.

We arrive at the following picture of the domain wall at hand. The quark fields vary from their VEV's $\sim \sqrt{\xi}$ to zero within small regions, of the order of $1 / \sqrt{\xi}$ (see the previous footnote). They remain small inside the wall, see Fig. 3.

Then to the leading order we can put the quark fields to zero in (5.6). Now, the second equation in (5.6) tells us that $a$ is a linear function of $z$. The solution for $a$ takes the form

$$
a=-\sqrt{2}\left(m-\Delta m \frac{z-z_{0}}{R}\right)
$$

where the collective coordinate $z_{0}$ is the position of the wall center (and $\Delta m$ is assumed positive). The solution is valid in a wide domain of $z$

$$
\left|z-z_{0}\right|<\frac{R}{2}
$$

except narrow areas of size $\sim 1 / \sqrt{\xi}$ near the edges of the wall at $z-z_{0}= \pm R / 2$.

Substituting the solution (5.13) in the second equation in (5.6) we get

$$
R=\frac{4 \Delta m}{g^{2} \xi}=\frac{2 \Delta m}{m_{\gamma}^{2}} .
$$




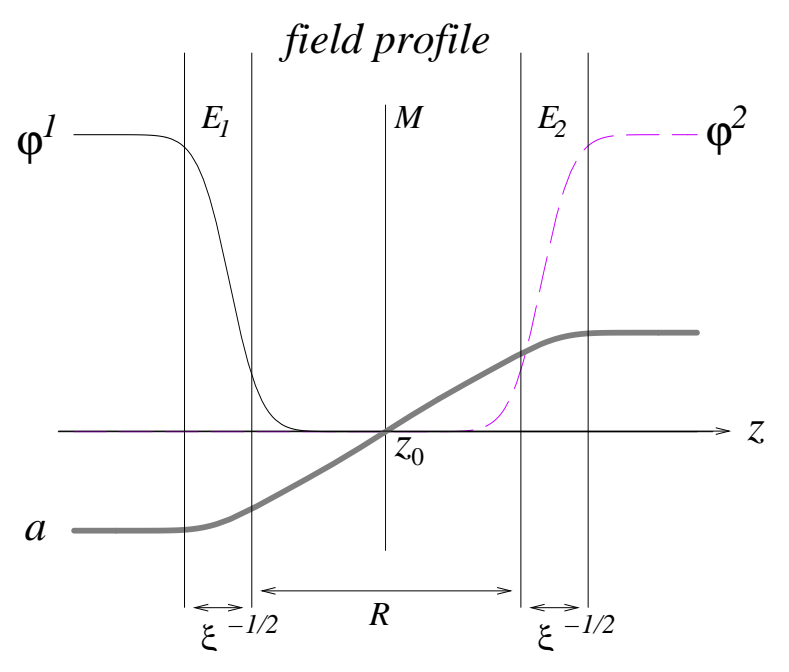

Figure 3: Internal structure of the domain wall: two edges (domains $E_{1,2}$ ) of the width $\sim \xi^{-1 / 2}$ are separated by a broad middle band (domain $M$ ) of the width $R$, see Eq. (5.15).

Since $\Delta m / \sqrt{\xi} \gg 1$, see Eq. (1.4), this result shows that $R \gg 1 / \sqrt{\xi}$, which justifies our approximation. It is easy to check that $1 / 2$ of the wall tension quoted in Eq. (5.8), comes from the kinetic term of the field $a$ in the middle domain $M$.

Furthermore, we can now use the first relation in Eq. (5.6) to determine tails of the quark fields inside the wall. First let us fix the gauge imposing the condition that $\varphi^{1}$ is real at $z \rightarrow-\infty$ and $\varphi^{2}$ is real at $z \rightarrow \infty$. This is a generalization of the unitary gauge for the problem with domain wall that interpolates between two vacua. Of course, this requirement does not fix the gauge completely. We still have freedom to make gauge transformations with gauge parameter which is non-zero inside the wall.

Let us assume that the gauge field is given by

$$
A_{z}=\sigma \partial_{z} \beta(z), \quad A_{n}=0, \quad n=0,1,2,
$$

so that the field strength is zero. Here $\beta(z)$ is some function of $z$ while $\sigma$ is a constant introduced in order to normalize $\beta(z)$ in a convenient way.

Consider first the left edge (domain $E_{1}$ in Fig. 3) at $z-z_{0}=-R / 2$. Substituting the above solution for $a$ in the equation for $\varphi^{1}$ we get

$$
\varphi^{1}=\sqrt{\xi} e^{-\frac{m_{\gamma}^{2}}{4}\left(z-z_{0}+\frac{R}{2}\right)^{2}+i \frac{\sigma}{2}[1+\beta(z)]}
$$

This behavior is valid in the domain $M$, at $\left(z-z_{0}+R / 2\right) \gg 1 / \sqrt{\xi}$, and shows that the field of the first quark flavor tends to zero exponentially inside the wall, as was expected. Our gauge choice requires

$$
\beta(z) \rightarrow-1, \quad z \rightarrow-\infty
$$


while inside the wall the function $\beta(z)$ remains undetermined reflecting the possibility of gauge transformations.

By the same token, we can consider the behavior of the second quark flavor near the right edge of the wall at $z-z_{0}=R / 2$. The first of equation in (5.6) for $A=2$ implies

$$
\varphi^{2}=\sqrt{\xi} e^{-\frac{m_{\gamma}^{2}}{4}\left(z-z_{0}-\frac{R}{2}\right)^{2}-i \frac{\sigma}{2}[1-\beta(z)]},
$$

which is valid in the domain $M$ provided that $\left(R / 2-z+z_{0}\right) \gg 1 / \sqrt{\xi}$. Inside the wall the second quark flavor tends to zero exponentially too. Our gauge choice implies that

$$
\beta(z) \rightarrow 1, \quad z \rightarrow \infty .
$$

Needless to say that the first and second quark flavor profiles are symmetric with respect to reflection at $z_{0}$. The potential term of the $\varphi^{A}$ fields in the domain $M$ produces the remaining $1 / 2$ of the wall tension,

$$
\int d z \frac{g^{2}}{8}\left(\left|\varphi^{A}\right|^{2}-\xi\right)^{2}=\frac{g^{2}}{8} \xi^{2} R=\frac{T_{\mathrm{w}}}{2} .
$$

This means of course that the contribution of the edges $E_{1,2}$ in the wall tension must be of higher order in $\xi$. With this remark we proceed to the edge domains.

In the domains near the wall edges, $z-z_{0}= \pm R / 2$, the fields $\varphi^{A}$ and $a$ smoothly interpolate between their VEV's in the given vacua and the behavior inside the wall determined by Eqs. (5.13) and (5.17), and (5.19). It is not difficult to check that these domains produce contributions to the wall tension of the order of $\xi^{3 / 2}$ which makes them negligibly small.

Now let us comment on the phase factors in (5.17), (5.19). Two complex fields $\varphi^{1}$ and $\varphi^{2}$ have, generally speaking, two independent phases. Since it is the diagonal $\mathrm{U}(1)$ that is gauged it is natural to parametrize them as a common phase which we denote as $\beta(z) \sigma / 2$ (it depends on the gauge transformations which we can still make inside the wall) and a relative phase $\sigma$. This phase $\sigma$ is nothing but a global $\mathrm{U}(1)$ remnant of the global flavor $\mathrm{SU}\left(N_{f}=2\right)$ symmetry which is explicitly broken down to $\mathrm{U}(1)$ due to the fact that $m_{1} \neq m_{2}$. It is worth stressing that $\sigma$ is a collective coordinate of the wall, rather than a modulus associated with the vacua. Because of the $\mathrm{U}(1) \times \mathrm{U}(1)$ symmetry of the Lagrangian (5.2), the effective theory on the wall has no potential energy associated with $\sigma$. At the same time, as was already mentioned, in the model under consideration there are no massless fields in the bulk — all fields are massive in each of two quark vacua.

Thus we have two collective coordinates characterizing our wall solution, the position of the center $z_{0}$ and the phase $\sigma$. In the effective low-energy theory on the wall they become scalar fields of the world-volume $2+1$ dimensional theory, $\zeta(t, x, y)$ and $\sigma(t, x, y)$, respectively. The target space of the second field is $S_{1}$, as is obvious from Eqs. (5.17) and (5.19). 


\subsection{Zero modes}

Two bosonic zero modes are obtained by differentiating the solution of Sec. 5.2 with respect to $z_{0}$ and $\sigma$. The first one is translational, the second can be called "rotational." Obtaining the translational zero mode is straightforward; it is depicted in Fig. 4, which gives an idea of its spread (localization). The rotational zero mode deserves a comment.

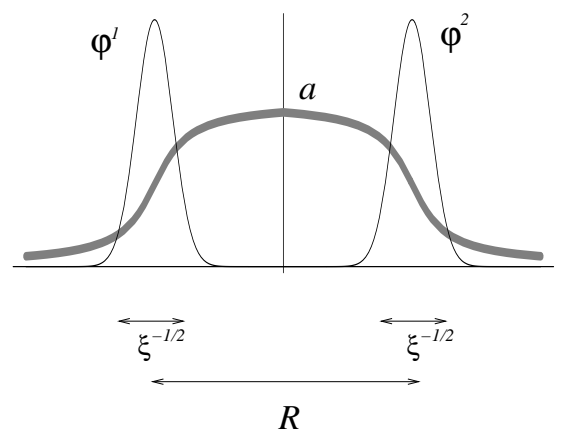

Figure 4: The translational zero mode.

The solution for the $a$ field is $\sigma$ independent. Therefore the rotational zero mode contains no $a$ component. As for the $\phi$ components, differentiating the solution with respect to $\sigma$ in a straightforward manner one obtains

$$
\varphi^{A}=\phi_{0}^{A}+\varphi_{\mathrm{zm}}^{A}, \quad A=1,2,
$$

where

$$
\begin{aligned}
\varphi_{\mathrm{zm}}^{1} & =\frac{\partial \varphi_{0}^{1}}{\partial \sigma} \delta \sigma=\frac{i}{2} \phi_{0}^{1}[1+\beta(z)] \delta \sigma \\
\varphi_{\mathrm{zm}}^{2} & =\frac{\partial \varphi_{0}^{2}}{\partial \sigma} \delta \sigma=-\frac{i}{2} \varphi_{0}^{2}[1-\beta(z)] \delta \sigma .
\end{aligned}
$$

Note that because of the boundary conditions (5.18), (5.20) these zero modes are normalizable. Qualitatively they are the same as the $\varphi$ modes in Fig. 4.

Now we will dwell on the fermion zero modes. There are fermion zero modes of two types, "supertranslational" and "super-rotational."

To generate these modes we apply supersymmetry transformations to the bosonic wall solution of Sec. 5.2. As we have already explained, four supercharges selected by conditions (5.12) act trivially on the wall solution. The remaining four supercharges act non-trivially giving fermion zero modes. To separate them we impose conditions between $\varepsilon^{\alpha f}$ and $\bar{\varepsilon}_{\dot{\alpha}}^{f}$ which differs by sign from those in Eq. (5.12). Namely, we take

$$
\begin{aligned}
& \bar{\varepsilon}_{\dot{2}}^{2}=i \varepsilon^{21}, \quad \bar{\varepsilon}_{\dot{2}}^{1}=i \varepsilon^{22}, \\
& \bar{\varepsilon}_{\dot{1}}^{1}=-i \varepsilon^{12}, \quad \bar{\varepsilon}_{\dot{1}}^{2}=-i \varepsilon^{11},
\end{aligned}
$$


and substitute this into Eq. (5.9). Using (5.6) we get the fermion zero modes on the wall

$$
\begin{aligned}
\lambda^{\alpha f} & =i \eta^{\alpha p}\left(\tau_{1}\right)_{p}^{f} g^{2}\left(\left|\varphi^{A}\right|^{2}-\xi\right), \quad f, p=1,2, \\
\psi^{\alpha A} & =i \eta^{\alpha f}\left(a+\sqrt{2} m_{A}\right) q_{f}^{A}, \\
\tilde{\psi}_{A}^{\alpha} & =i \eta^{\alpha f}\left(a+\sqrt{2} m_{A}\right) \bar{q}_{f A},
\end{aligned}
$$

where by definition

$$
q_{f}^{A}=\frac{1}{\sqrt{2}}\left(\varphi^{A},-\varphi^{A}\right), \quad \bar{q}_{f A}=\frac{1}{\sqrt{2}}\left(\bar{\varphi}_{A}, \bar{\varphi}_{A}\right),
$$

while $\eta^{\alpha f}$ are four Grassmann parameters parametrizing the fermion zero modes. Under the supertransformations

$$
\delta \eta^{\alpha f}=\varepsilon^{\alpha f}
$$

Here the fields $a$ and $\varphi^{A}$ are given by Eqs. (5.13) and (5.17), (5.17). Note that the conjugated fermion fields are given by conjugation of Eqs. (5.25), however the parameters $\bar{\eta}_{\dot{\alpha}}^{f}$ are not independent. They are expressed in terms of $\eta^{\alpha f}$ according to

$$
\begin{aligned}
& \bar{\eta}_{\dot{2}}^{2}=i \eta^{21}, \quad \bar{\eta}_{\dot{2}}^{1}=i \eta^{22}, \\
& \bar{\eta}_{\dot{1}}^{1}=-i \eta^{12}, \quad \bar{\eta}_{\dot{1}}^{2}=-i \eta^{11} .
\end{aligned}
$$

We see that the $\lambda$ fermions (i.e. photino plus its $\mathcal{N}=2$ partner) are non-zero and approximately constant inside the wall while the matter fermions $\psi$ are concentrated near the wall edges. This picture is a super-reflection of that in Fig. 4.

\subsection{Effective field theory on the wall}

In this subsection we work out $(2+1)$-dimensional effective low-energy theory of the moduli on the wall. To do so we make the wall collective coordinates $z_{0}$ and $\sigma$ (together with their fermionic superpartners $\eta^{\alpha f}$ ) slowly varying fields depending on $x_{n}(n=0,1,2)$,

$$
z_{0} \rightarrow \zeta\left(x_{n}\right), \quad \sigma \rightarrow \sigma\left(x_{n}\right), \quad \eta^{\alpha f} \rightarrow \eta^{\alpha f}\left(x_{n}\right) .
$$

For simplicity let us consider the bosonic fields $\zeta\left(x_{n}\right)$ and $\sigma\left(x_{n}\right)$; the residual supersymmetry will allow us to readily reconstruct the fermion part of the effective action.

Because $\zeta\left(x_{n}\right)$ and $\sigma\left(x_{n}\right)$ correspond to zero modes of the wall, they have no potential terms in the world sheet theory. Therefore, in fact our task is to derive 
kinetic terms. For $\zeta\left(x_{n}\right)$ this procedure is very simple. Substituting the wall solution (5.13), (5.17), and (5.19) in the action (5.2) and taking into account the $x_{n}$ dependence of this modulus we immediately get (cf. (2.8))

$$
\frac{T_{\mathrm{w}}}{2} \int d^{3} x\left(\partial_{n} \zeta\right)^{2}
$$

As far as the kinetic term for $\sigma\left(x_{n}\right)$ is concerned more effort is needed. We start from Eqs. (5.17) and (5.19) for the quark fields ${ }^{5}$. Then we will have to modify our ansatz for the gauge field ${ }^{6}$,

$$
A_{\mu}=\partial_{\mu}\left[\sigma\left(x_{n}\right) \beta(z)\right]+\chi(z) \partial_{\mu} \sigma\left(x_{n}\right) .
$$

A few important points to be noted are as follows.

(i) We have introduced an extra profile function $\chi(z)$. It has no role in the construction of the static wall solution per se. It is unavoidable, however, in constructing the kinetic part of the world sheet theory of the moduli. This new profile function is described by its own action, which will be subject to minimization. This seems to be an element of the procedure which is sufficiently general (previously a similar construction was applied e.g. in Ref. [32]), and yet, to the best of our knowledge, no proper coverage can be found in the literature.

(ii) The first term in Eq. (5.29) is pure gauge; it replaces a similar term in Eq. (5.16) where $\sigma$ was $x$ independent. The function $\beta(z)$ remains undetermined, and so does the pure gauge term. This should not worry us since they do not show up in any physical observables. The second term in Eq. (5.29), on the other hand, is not pure gauge. It does lead to a non-vanishing field strength. It is introduced in order to cancel the $x$ dependence of the quark fields far from the wall (in the quark vacua at $z \rightarrow \pm \infty$ ) emerging through the $x$ dependence of $\sigma\left(x_{n}\right)$, see Eqs. (5.17) and (5.19).

To ensure this cancellation we impose the following boundary conditions for the function $\chi(z)$

$$
\chi(z) \rightarrow \mp 1, z \rightarrow \pm \infty
$$

This parallels the procedure outlined in the toy model of Sect. 2. Next, substituting (5.17), (5.19) and (5.29) in the action (5.2) we arrive at

$$
\begin{aligned}
S_{2+1}^{\sigma} & =\left[\int d^{3} x \frac{1}{2}\left(\partial_{n} \sigma\right)^{2}\right] \\
& \times \int d z\left\{\frac{1}{g^{2}}\left(\partial_{z} \chi\right)^{2}+(1-\chi)^{2}\left|\varphi^{1}\right|^{2}+(1+\chi)^{2}\left|\varphi^{2}\right|^{2}\right\} .
\end{aligned}
$$

\footnotetext{
${ }^{5}$ Strictly speaking, these equations per se are valid only inside the wall, in the domain $M$. Outside the wall $\varphi^{1} \rightarrow \sqrt{\xi} \exp [(i \sigma / 2)(1+\beta(z))]$ at $z \rightarrow-\infty$ and $\varphi^{2} \rightarrow \sqrt{\xi} \exp [(-i \sigma / 2)(1-\beta(z))]$ at $z \rightarrow \infty$.

${ }^{6}$ Remember the electric charge of the quark fields is $\pm 1 / 2$.
} 
The expression in the second line is an "action" for the $\chi$ profile function. To get the classical solution for the BPS wall and the wall world volume the theory of moduli we will have to minimize this "action."

The last two terms in the braces - the potential terms in the action for $\chi$ come from the kinetic terms for the quark fields. The first term $\left(\partial_{z} \chi\right)^{2}$ in the braces - the kinetic term in the action for $\chi$ - comes from the kinetic term of the gauge field. Indeed, the second term in Eq. (5.29) produces a field strength,

$$
F_{z n}=\partial_{z} \chi \partial_{n} \sigma
$$

This field strength gives rise to the kinetic term for $\chi$ in the action (5.31).

Now to find the function $\chi$ we have to minimize (5.31) with respect to $\chi$. This gives the following equation:

$$
-\partial_{z}^{2} \chi-g^{2}(1-\chi)\left|\varphi^{1}\right|^{2}+g^{2}(1+\chi)\left|\varphi^{2}\right|^{2}=0 .
$$

Note, that the equation for $\chi$ is of the second order. This is because the domain wall is no longer BPS state once we switch on the dependence of the moduli on the "longitudinal" variables $x_{n}$.

To the leading order in $\sqrt{\xi} / \Delta m$ the solution of Eq. (5.33) can be obtained in the same manner as we did previously for other profile functions. Let us first discuss what happens outside the inner part of the wall. Say, at $z-z_{0} \gg R / 2$ the profile $\left|\varphi^{1}\right|$ vanishes while $\left|\varphi^{2}\right|$ is exponentially close to $\sqrt{\xi}$ and, hence,

$$
\chi \rightarrow-1+\text { const } e^{-m_{\gamma}\left(z-z_{0}\right)} .
$$

The picture at $z_{0}-z \gg R / 2$ is symmetric, with the interchange $\varphi^{1} \leftrightarrow \varphi^{2}$. Thus, outside the inner part of the wall, at $\left|z-z_{0}\right| \gg R / 2$, the function $\chi$ approaches its boundary values \pm 1 with the exponential rate of approach.

Of most interest, however, the inside part, the middle domain $M$ (see fig. 3). Here both quark profile functions vanish, and Eq. (5.33) degenerates into $\partial_{z}^{2} \chi=0$. As a result, the solution takes the form

$$
\chi=-2 \frac{z-z_{0}}{R}
$$

In narrow edge domains $E_{1,2}$ the exact $\chi$ profile smoothly interpolates between the boundary values, see Eq. (5.34), and the linear behavior (5.35) inside the wall. These edge domains give small corrections to the leading term in the action.

Substituting the solution (5.35) in the $\chi$ action, the second line in Eq. (5.31), we finally arrive at

$$
S_{2+1}^{\sigma}=\frac{\xi}{\Delta m} \int d^{3} x \frac{1}{2}\left(\partial_{n} \sigma\right)^{2}
$$

As has been already mentioned previously, the compact scalar field $\sigma(t, x, y)$ can be reinterpreted to be dual to the $(2+1)$-dimensional Abelian gauge field living on 
the wall, see Eq. (1.5). The emergence of the gauge field on the wall is easy to understand. The quark fields almost vanish inside the wall. Therefore the U(1) gauge group is restored inside the wall while it is higgsed in the bulk. The dual $\mathrm{U}(1)$ is in the confinement regime in the bulk. Hence, the dual $\mathrm{U}(1)$ gauge field is localized on the wall, in full accordance with the general argument of Ref. [2]. The compact scalar field $\sigma\left(x_{n}\right)$ living on the wall is a manifestation of this magnetic localization.

The result in Eq. (5.36) implies that the coupling constant of our effective U(1) theory on the wall is given by

$$
e_{2+1}^{2}=4 \pi^{2} \frac{\xi}{\Delta m} .
$$

In particular, the definition of the $(2+1)$-dimensional gauge field (1.5) takes the form

$$
F_{n m}^{(2+1)}=\frac{e_{2+1}^{2}}{2 \pi} \varepsilon_{n m k} \partial^{k} \sigma
$$

This finally leads us to the following effective low-energy theory of the moduli fields on the wall:

$$
S_{2+1}=\int d^{3} x\left\{\frac{T_{w}}{2}\left(\partial_{n} \zeta\right)^{2}+\frac{1}{4 e_{2+1}^{2}}\left(F_{n m}^{(2+1)}\right)^{2}+\text { fermion terms }\right\} .
$$

The fermion fields living on the wall are associated with the four fermion moduli $\eta^{\alpha f}$. On the grounds of $(2+1)$-dimensional Lorentz symmetry on the wall we may be certain that these four fermion moduli fields form two (two-component) Majorana spinors. Thus, the field content of the world sheet theory we have obtained is in full accord with the representation of $(2+1)$-dimensional extended supersymmetry (i.e. that with four supercharges ${ }^{7}$ ).

Now let us address the question as to the relative magnitude of the $(2+1)$ dimensional gauge coupling. We expect that the lightest massive excitations on the wall have mass of the order of the inverse wall thickness $\sim 1 / R$. Since

$$
\frac{1}{R}=\frac{g^{2}}{4} \frac{\xi}{\Delta m}=e_{2+1}^{2} \frac{g^{2}}{16 \pi^{2}},
$$

our $(2+1)$-dimensional coupling constant (5.37) is large in this scale, so that the theory on the wall is in the strong coupling regime. This could have been expected since it is a $\mathrm{U}(1)$ theory of the dual degrees of freedom (magnetic charges), as will be discussed in more detail in Sect. 7 .

\subsection{Nonzero modes}

It is not difficult to see that the lightest massive excitations are associated with perturbation of the size of the middle domain $M$. When its thickness "breathes," this gives rise to the softest mode. The mass of the softest mode can be readily estimated to be of the order of $m_{\gamma}^{2} / \Delta m \sim R^{-1}$.

\footnotetext{
${ }^{7}$ Minimal supersymmetric theories in $2+1$ dimensions have 2 supercharges.
} 


\section{6 $\mathcal{N}=2$ supersymmetry and the multiplicity of the do- main walls}

We have just demonstrated that one can find 1/2 BPS saturated domain wall in the $\mathcal{N}=2$ SQED with the generalized Fayet-Iliopoulos term. This fact per se has far reaching consequences for the world-volume theory as well as for the multiplicity of the domain walls. Indeed, $2+1$ dimensional world-volume theory must have four supercharges. This implies with necessity that there are two (massless) boson fields in the world-volume theory. On general grounds they can form either a chiral supermultiplet of $\mathcal{N}=2$ in $1+2$ or a vector supermultiplet of a world-volume theory with $\mathrm{U}(1)$ gauge invariance (linear supermultiplet). As long as the fields are massless, there is a duality between these two descriptions.

Supersymmetrization requires two (Majorana) two-component fields in the worldvolume theory. The dimension of the supermultiplet we deal with is four. The question to be discussed is: how many distinct domain walls we have? This question is meaningful in light of the recent finding $[24,12,13]$ of nontrivial multiplicity of the domain walls in $\mathcal{N}=1$ super-Yang-Mills theories.

In addressing this question we have to explain our convention. Every $(2+1)$ dimensional domain wall emerging in the $(3+1)$-dimensional theory has a translational and supertranslational moduli. If we quantize these moduli in a finite volume the corresponding wall multiplicity is two. This part of the moduli dynamics is trivial, however, and can (and should) be factored out. That's what we will always do. When we speak of the wall multiplicity we discard the above trivial degeneracy and focus exclusively on possible extra degeneracy not associated with the (super)translational moduli. With this convention one can demonstrate $[24,12,13]$ that the number of distinct domain walls in $\mathrm{SU}(N)$ super-Yang-Mills theories is in fact $N$.

Following this convention we can say that the $1 / 2$ BPS wall we have constructed has multiplicity two. This is rather obvious by itself, since in the case at hand the original $(3+1)$-dimensional theory we began with had eight supercharges. What is remarkable is that the above statement will hold even if we break $\mathcal{N}=2$ of the original theory down to $\mathcal{N}=1$.

To see that the wall multiplicity is two it is sufficient to compactify the longitudinal directions $x$ and $y$. Then the reduced moduli field theory on the wall ${ }^{8}$ becomes quantum mechanics of one real variable $\sigma(t)$ defined on a circle,

$$
\sigma+2 \pi \leftrightarrow \sigma
$$

and two fermion variables $\psi(t)$ and $\bar{\psi}(t)$. This supersymmetric quantum mechanics has the ground state at zero energy which is doubly degenerate. This double degeneracy is protected against nonsingular perturbations, such as generation of the potential term for $\sigma$, which, generally speaking, might occur if $\mathcal{N}=2$ is broken down

\footnotetext{
${ }^{8}$ By reduced we mean that the translational modulus and its superpartner are factored out.
} 
to $\mathcal{N}=1$. The potential term is not generated for our domain wall; presumably, it is generated in the case considered in Ref. [23].

\section{The ANO strings}

In string theory gauge fields are localized on D branes because fundamental open strings can end on a D brane. Our task now is to investigate to which extend this picture - a flux tube ending on the critical domain wall - holds in field theory. We will see that the answer to this question is positive: our $1 / 2$ BPS domain wall does allow for the magnetic flux tubes to end on it.

As we have already explained, both quark vacua in our $\mathcal{N}=2$ QCD give rise to confinement phase for monopoles [5, 16]. The monopoles themselves are very heavy in the quark vacua; the monopole mass is of the order of $\mathrm{m} / \mathrm{g}^{2}$. Hence, the monopoles can be considered as probes for confinement. Here we deal with the Abelian confinement, due to the ANO flux tubes which stretch between monopoles and antimonopoles.

Now we will demonstrate, through an exact solution, that the ANO string can end on the domain wall interpolating between two quark vacua. Imagine a monopole placed at some point in the bulk far away from the domain wall. The magnetic flux of the monopole is trapped inside the flux tube in the bulk. When the tube joins the wall the magnetic flux of the tube becomes electric flux of the dual U(1) theory on the wall; therefore it spreads out along the wall. The end point of the tube on the wall plays the role of an electric charge in the $(2+1)$-dimensional $\mathrm{U}(1)$ gauge theory. Ending the flux tube on the wall, rather than letting the tube go through, is energetically advantageous. The field configuration with a string attached to the wall is $1 / 4$ BPS saturated in our model. In other words, the theory localized on the string-wall junction has two supercharges.

Before delving in details of the string-wall junction construction (which will be done shortly) we have to briefly review the critical ANO strings in the SeibergWitten theory $[18,16,19,6,20]$. Our presentation follows that of Ref. [6], the only difference is that Ref. [6] deals with the critical strings in the monopole/dyon vacua while here we are interested in the quark vacua.

Let us consider, say, the ANO strings in the vacuum (4.6). The field $a$ is irrelevant for the string solution so we can put it equal to its VEV (4.6) and drop from the effective QED Lagrangian, Eq. (4.2). In the vacuum (4.6) the second quark flavor $q^{2}$ is heavier than the first one and we can ignore it either. Moreover, it turns out that the string solution we are after admits the same ansatz (5.1) we exploited for finding the wall solution.

With all these simplifications the effective action of our model (5.2) becomes

$$
S_{\mathrm{str}}=\int d^{4} x\left\{\frac{1}{4 g^{2}} F_{\mu \nu}^{2}+\left|\nabla_{\mu} \varphi^{1}\right|^{2}+\frac{g^{2}}{8}\left(\left|\varphi^{1}\right|^{2}-\xi\right)^{2}\right\} .
$$


First, let us compare this theory with the general Abelian Higgs model in which the ANO vortices are known to exist [17]. The action of the Abelian Higgs model reads

$$
S_{\mathrm{AH}}=\int d^{4} x\left\{\frac{1}{4 g^{2}} F_{\mu \nu}^{2}+\left|\nabla_{\mu} \varphi^{1}\right|^{2}+\lambda\left(\left|\varphi^{1}\right|^{2}-\xi\right)^{2}\right\} .
$$

We see that the model (6.1) which appears in $\mathcal{N}=2$ QED with the FI term (the same model appears in $\mathcal{N}=1$ SUSY with the FI term [30]) corresponds to a special value of the coupling $\lambda$,

$$
\lambda=\frac{g^{2}}{8} .
$$

In the model (6.2) photon has mass (4.10) while quark $\varphi^{1}$ acquires the mass

$$
m_{H}^{2}=4 \lambda \xi .
$$

For generic values of $\lambda$ in Eq. (6.2) the quark mass (the inverse correlation length) and the photon mass (the inverse penetration depth) are distinct. Their ratio is an important parameter characterizing the type of the superconductor under consideration. Namely, for $m_{H}<m_{\gamma}$ one deals with the type I superconductor in which two strings at large separations attract each other. On the other hand, for $m_{H}>m_{\gamma}$ the superconductor is of type II, in which case two strings at large separation repel each other. This behavior is related to the fact that the scalar field generates attraction between two vortices, while the electromagnetic field generates repulsion.

Now we see that with the choice (6.3) for $\lambda$ the masses are equal,

$$
m_{\gamma}=m_{H}
$$

and the superconductor is exactly at the border between type I and type II. The relation (6.5) has important consequences. It means that the ANO string satisfies the first order equations and saturates the Bogomolny bound [26]. The BPS strings do not interact.

To get the BPS equations for the string in the model (6.1) (as well as the BPS bound for its tension) it is convenient to perform the Bogomolny completion of the action,

$$
\begin{aligned}
T_{\text {str }} & =\int d^{2} x\left\{\frac{1}{2 g^{2}}\left[F_{3}^{*}-\frac{g^{2}}{2}\left(\left|\varphi^{1}\right|^{2}-\xi\right)\right]^{2}+\right. \\
& \left.+\left|\left(\nabla_{1}-i \nabla_{2}\right) \varphi^{1}\right|^{2}\right\}+2 \pi \xi n .
\end{aligned}
$$

Here we assume that the string is aligned along the $x_{3}=z$ axis with its center at the point $x_{1}=x_{2}=0$ and, moreover,

$$
F_{n}^{*}=\frac{1}{2} \varepsilon_{n m k} F_{m k}, \quad n, m, k=1,2,3 .
$$


The last term in (6.6) measures the quantized magnetic flux of the vortex, $n$ is the winding number. For simplicity we will consider the minimal winding, $n=1$.

As usual, implications of the Bogomolny completion are immediate. The energy minimum is reached if the first two terms in the braces vanish individually. Then the string tension is, obviously,

$$
T_{\text {str }}=2 \pi \xi
$$

The vanishing of the first two terms in the braces imply the following first order equations:

$$
\begin{aligned}
& F_{3}^{*}-\frac{g^{2}}{2}\left(\left|\varphi^{1}\right|^{2}-\xi\right)=0, \\
& \left(\nabla_{1}-i \nabla_{2}\right) \varphi^{1}=0 .
\end{aligned}
$$

Certainly, these equations are well studied in the literature [26].

The classical ANO vortex solution for the fields $\varphi^{1}$ and $A_{\mu}$ is obtained in the standard ansatz,

$$
\begin{aligned}
\varphi^{1}(x) & =\phi(r) e^{-i \alpha} \\
A_{i}(x) & =2 \varepsilon_{i j} \frac{x_{j}}{x^{2}}[1-f(r)],
\end{aligned}
$$

where $i, j=1,2, r=\sqrt{x_{i}^{2}}$ and $\alpha$ is the polar angle in the (1,2) plane (Fig. 1$)$. The real profile functions $\phi(r)$ and $f(r)$ satisfy the boundary conditions

$$
\begin{aligned}
& \phi(0)=0, \quad f(0)=1, \\
& \phi(\infty)=\sqrt{\xi}, \quad f(\infty)=0,
\end{aligned}
$$

which ensure that the scalar field reaches its $\mathrm{VEV} \sqrt{\xi}$ at infinity and the vortex carries precisely one unit of the magnetic flux.

With this ansatz, the first order equations (6.9) become

$$
\begin{aligned}
& \phi^{\prime}-\frac{1}{r} f \phi=0, \\
& -\frac{1}{r} f^{\prime}+\frac{g^{2}}{4}\left(\phi^{2}-\xi\right)=0 .
\end{aligned}
$$

These equations, together with the boundary conditions (6.11), can be solved numerically.

In supersymmetric theories the Bogomolny bound (6.8) for the string tension can be viewed as a central charge (in the anticommutator $\left\{Q_{\alpha}, \bar{Q}_{\dot{\beta}}\right\}$ ) of the supersymmetry algebra. The first order equations (6.9) can be obtained by requiring half 
of the supercharges to act trivially on the string solution $[29,30,31,6]$. In order to see which particular supercharges act trivially we write down all relevant SUSY transformations (5.9) in our effective QED and put the fermion components to zero. Dropping the fields $a$ and $q^{2}$ (as was discussed in the beginning of this section) and assuming that all fields depend only on the coordinates $x_{1}, x_{2}$ we arrive at the same equations (6.9) provided that the SUSY transformation parameters $\varepsilon^{\alpha f}$ and $\bar{\varepsilon}_{\dot{\alpha}}^{f}$ are subject to the following constraints:

$$
\begin{array}{ll}
\varepsilon^{12}=-\varepsilon^{11}, & \bar{\varepsilon}_{\dot{1}}^{2}=-\bar{\varepsilon}_{\dot{1}}^{1}, \\
\varepsilon^{21}=\varepsilon^{22}, & \bar{\varepsilon}_{\dot{2}}^{1}=\bar{\varepsilon}_{\dot{2}}^{2} .
\end{array}
$$

These conditions select those supercharges which act trivially on the BPS string solution. Moreover, they explicitly show that our ANO string is 1/2 BPS-saturated. Note, however, that the conditions (6.13) are different from those in Eq. (5.12), so that four supercharges preserved by the string and four supercharges preserved by the wall are not the same.

\section{String ending on the wall}

In this section we derive BPS equations and find a $1 / 4$ BPS solution for the wallstring junction. We analyze qualitative features of the solution and investigate how the magnetic flux of the string gets spread inside the wall.

\subsection{First order equations for a string ending on the wall}

It is natural to assume that at large distances from the string end point at $r=0$, $z=0$, the wall is almost parallel to the $\left(x_{1}, x_{2}\right)$ plane while the string is stretched along the $z$ axis, see Fig. 1. Since both solutions, for the string and the wall, were obtained using the ansatz (5.1) we restrict our search for the wall-string junction to the same ansatz. As usual, we look for a static solution assuming that all relevant fields can depend only on $x_{n},(n=1,2,3)$.

First, we have to decide which particular combinations of supercharges act trivially on the wall-string junction configuration. To this end we impose both, the wall conditions (5.12) and the string conditions (6.13), simultaneously. It turns out that there is a nontrivial solution with

$$
\begin{aligned}
& \varepsilon^{12}=-\varepsilon^{11}, \\
& \varepsilon^{21}=\varepsilon^{22},
\end{aligned}
$$

while the parameters $\bar{\varepsilon}$ are given in Eq. (5.12). We see that all eight SUSY parameters can be expressed in terms of two arbitrary parameters $\varepsilon^{11}$ and $\varepsilon^{22}$. Thus, the string-ending-on-the-wall configuration is $1 / 4$ BPS saturated. 
Now we substitute Eq. (7.1) in the SUSY transformations (5.9) and put the fermion components to zero. This leads us to the following first order equations:

$$
\begin{aligned}
& F_{1}^{*}-i F_{2}^{*}-\sqrt{2}\left(\partial_{1}-i \partial_{2}\right) a=0 \\
& F_{3}^{*}-\frac{g^{2}}{2}\left(\left|\varphi^{A}\right|^{2}-\xi\right)-\sqrt{2} \partial_{3} a=0 \\
& \nabla_{3} \varphi^{A}=-\frac{1}{\sqrt{2}} \varphi^{A}\left(a+\sqrt{2} m_{A}\right) \\
& \left(\nabla_{1}-i \nabla_{2}\right) \varphi^{A}=0
\end{aligned}
$$

These equations generalize the first order equations for the wall and for the string $\left(F^{*}\right.$ is defined in Eq. (6.7)).

It is instructive to check that both, the wall and the string solutions, separately, satisfy these equations. Start from the wall. In this case the gauge field is pure gauge (see Eq. (5.16)), and all fields depend only on $z$. Thus, the first and the last equations in (7.2) are trivially satisfied. The component of the gauge field $F_{3}^{*}$ vanishes in the second equation and this equation reduces to the second equation in Eq. (5.6). The third equation in (7.2) coincides with the first one in (5.6).

For the string which lies, say, in the vacuum (4.6), the second quark flavor vanishes, $q^{2}=0$, while $a$ is given by its $\mathrm{VEV}$. The electromagnetic flux is directed along the $z$ axis, so that $F_{1}^{*}=F_{2}^{*}=0$. All fields depend only on the coordinates $x_{1}$ and $x_{2}$. Then the first and the third equations in (7.2) are trivially satisfied. The second equation reduces to the first one in Eq. (6.9). The last equation in (7.2) for $A=1$ reduces to the second equation in (6.9), while for $A=2$ this equation is trivially satisfied, quod erat demonstrandum.

\subsection{The string-wall junction (Solution for a string ending on the wall)}

Needless to say that the solution of first order equations (7.2) for a string ending on the wall can be found only numerically especially near the end point of the string where both the string and the wall profiles are heavily deformed. However, far away from the end point of the string, deformations are weak and we can find the asymptotic behavior analytically.

Let the string be on the $z>0$ side of the wall, where the vacuum is given by Eq. (4.7), see Fig. 1. Consider first the region $z \rightarrow \infty$ far away from the string end point at $z \sim 0$. Then the solution to (7.2) is given by an almost unperturbed string. Namely, at $z \rightarrow \infty$ there is no $z$ dependence to the leading order, and, hence, the following ansatz for fields $A_{1,2}$ and $\varphi^{2}$ is appropriate:

$$
\varphi^{2}(x)=\phi(r)
$$




$$
A_{i}(x)=-\varepsilon_{i j} \frac{x_{j}}{x^{2}} f(r)
$$

It differs from the one in Eq. (6.10) by a gauge transformation, to the "singular" gauge, in which the scalar field $\varphi^{2}$ is aligned along its VEV at $r \rightarrow \infty, z \rightarrow \infty$. The profile functions $\phi(r)$ and $f(r)$ satisfy the boundary conditions (6.11). We also take the fields $A_{3}, A_{0}$ and $\varphi^{1}$ to be zero, with $a$ equal to its VEV (4.7). Then Eqs. (7.2) reduce to those of (6.12). The latter have a standard solution of the unperturbed ANO string. On the other side of the wall, at $z \rightarrow-\infty$, we have an almost unperturbed first vacuum with the fields given by their VEV's (4.6) and (4.8).

Now consider the domain $r \rightarrow \infty$ at small $z$. In this domain the solution to (7.2) is given by a perturbation of the wall solution. Let us use the ansatz in which the solutions for the fields $a$ and $q^{A}$ are given by the same equations (5.13), (5.17) and (5.19) in which the size of the wall is still given by (5.15), and the only modification is that the position of the wall $z_{0}$ and the phase $\sigma$ now become slowly-varying functions of $r$ and $\alpha$ (i.e. the polar coordinates on the $\left(x_{1}, x_{2}\right)$ plane). It is quite obvious that $z_{0}$ will depend only on $r$, as schematically depicted in Fig. 5. The physical meaning of this "adiabatic" approximation is as follows: the massive excitations of the wall, responsible for its structure, are assumed to be absent; we study the impact of the string-wall junctions on the massless moduli.

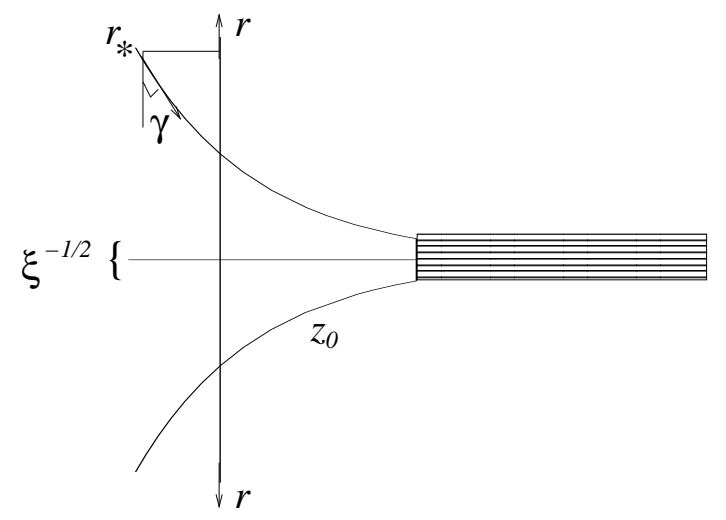

Figure 5: Bending of the wall due to the string-wall junction. The flux tube extends to the right infinity. The wall profile is logarithmic at transverse distances larger than $\xi^{-1 / 2}$ from the string axis. At smaller distances the adiabatic approximation fails.

As long as the second and the third equations in (7.2) do not contain derivatives with respect to $x_{i}, i=1,2$, they are satisfied identically for any functions $z_{0}(r, \alpha)$ and $\sigma(r, \alpha)$ (note, that $F_{3}^{*}=0$, the field strength is parallel to the domain wall plane and $A_{z}=\sigma(r, \alpha) \partial_{z} \beta(z)$, see Eq. (5.16)). 
However, the first and the last equations in (7.2) become nontrivial. Consider the first one. Inside the string the electromagnetic field is directed along the $z$ axis and its flux is given by $4 \pi$. This flux is spread out inside the wall and directed almost along $x_{i}$ in the $\left(x_{1}, x_{2}\right)$ plane at large $r$. Since the flux is conserved, we have

$$
F_{i}^{*}=\frac{2}{R} \frac{x_{i}}{r^{2}}
$$

inside the wall at $\left|z-z_{0}(r, \alpha)\right|<R / 2$.

Substituting this in the first equation in (7.2) and assuming that $z_{0}$ depends only on $r$ we then get

$$
\partial_{r} z_{0}=-\frac{1}{\Delta m r}
$$

Needless to say that our adiabatic approximation holds only provided the above derivative is small, i.e. sufficiently far from the string, $\sqrt{\xi} r \gg 1$.

The solution to this equation is straightforward,

$$
z_{0}=-\frac{1}{\Delta m} \ln r+\text { const. }
$$

We see that the wall is logarithmically bent according to the Coulomb law in $2+1$ dimensions $^{9}$ (see Fig. 5). This bending produces a balance of forces between the string and the wall in the $z$ direction so that the whole configuration is static. To see that this is indeed the case, please, observe that the force of the string is equal to the string tension $2 \pi \xi$ (see Eq. (6.8)). On the other hand, the force of the wall in $z$-direction at some point $z_{*}$ is given by the wall tension $\xi \Delta m$ times the length of the circle $2 \pi r_{*}\left(r_{*}\right.$ corresponds to $z_{*}$ via Eq. (7.6)) times the angle $\gamma$ following 10 from Eq. (7.5), which projects the force of the bent wall onto the $z$ axis. This gives $2 \pi \xi$, which precisely coincides with the string tension.

Now let us consider the last equation in (7.2). First, let us work out the gauge potential which enters the covariant derivatives in this equation. In order to produce the field strength (7.4) $A_{\mu}$ should reduce to

$$
\begin{aligned}
& A_{i}=\frac{2}{R} \varepsilon_{i j} \frac{x_{j}}{r^{2}}\left[z-z_{0}(r)\right]+\beta(z) \partial_{i} \sigma\left(x_{j}\right), \quad i=1,2, \\
& A_{0}=0, \quad A_{z}=\sigma\left(x_{i}\right) \partial_{z} \beta(z)
\end{aligned}
$$

where we also include pure gauge terms, see Eq. (5.16). Consider first the region near the edge of the wall at $z-z_{0} \sim-R / 2$. Near this edge the first quark field $\varphi^{1}$ is not zero. Substituting (5.17) in the last equation in (7.2) and using (7.7) and (7.5) we get

$$
\frac{\partial \sigma}{\partial \alpha}=1, \quad \frac{\partial \sigma}{\partial r}=0 .
$$

\footnotetext{
${ }^{9}$ The logarithmic bending in two spatial dimensions was used by Witten to explain logarithmic running of coupling constant of $\mathcal{N}=2$ theories within the brane approach [35].

${ }^{10}$ One should remember that $r_{*} \gg(\Delta m)^{-1}$, so that $\gamma \approx \tan \gamma \approx \sin \gamma$.
} 
The solution to these equations is

$$
\sigma=\alpha .
$$

This vortex solution is certainly expected and welcome. In terms of the dual gauge field localized on the wall, this solution reflects nothing but the unit source charge. (If we consider the other edge of the wall near $z-z_{0} \sim R / 2$ and substitute (5.19) into the last equation in (7.2) we get the same equations $(7.8)$ for $\sigma(r, \alpha)$.)

The above relation between the vortex solution and the unit source charge requires a comment. One can identify the compact scalar field $\sigma$ with the electric field living on the domain wall world volume via (5.38). Then result (7.9) gives (1.7) for this electric field. In the proper normalization we have

$$
F_{0 i}^{(2+1)}=\frac{e_{2+1}^{2}}{2 \pi} \frac{x_{i}}{r^{2}}
$$

where the $(2+1)$-dimensional coupling is given by (5.37).

This is a field of a point-like electric charge in $2+1$ dimensions placed at $x_{i}=0$. The interpretation of this result is that the string end point on the wall plays a role of the electric charge in the dual $\mathrm{U}(1)$ theory on the wall.

Our string-wall junction solution explicitly demonstrates another rather apparent aspect of the problem at hand. Indeed, when the string ends on the wall, and the magnetic flux it brings with it spreads out inside the wall, the overall energy of the configuration is minimized. Indeed, the energy of the flux tube grows linearly with its dimension, while when the flux is spread inside the wall, the energy grows only logarithmically with the dimension of the domain over which the flux is spread. It is certainly no accident that the wall bending is logarithmic.

\section{Flow to $\mathcal{N}=1$ theory}

It is high time now to address the question what happens with the moduli theory once we include subleading in $\mu$ terms that break $\mathcal{N}=2$ supersymmetry down to $\mathcal{N}=1$. At the level of the low-energy QED (4.2) to which we limit ourselves in the present paper this amounts to taking into account the $a$ dependence of the function $f(a)$ in Eq. (4.2),

$$
f(a) \equiv-2 \sqrt{2} \mu \frac{\partial u}{\partial a}=-2 \sqrt{2} \mu a
$$

while previously $f(a)$ was set to be constant, $f(a)=\xi$, in which limit we deal with the fully blown $\mathcal{N}=2$.

Let us first discuss the value of an $\mathcal{N}=2$ breaking parameter on general grounds. It is quite clear that in both vacua this parameter can be estimated as

$$
\frac{\mu \delta a_{\text {char }}}{\xi} \sim \frac{\mu \sqrt{\xi}}{\xi} \sim \sqrt{\frac{\mu}{m}}
$$


This estimate is confirmed, in particular, by the calculation of the fermion mass shift presented in Sect. 8.2. However when one deals with collective excitations of the domain wall (other than the zero modes), then the $\mathcal{N}=2$ breaking parameter is larger, since in this case $\delta a_{\text {char }}$ is of the order of $\Delta m$. Then, the $\mathcal{N}=2$ breaking parameter is of the order of

$$
\frac{\mu \Delta m}{\xi} .
$$

Although this is still a small parameter it is nevertheless larger than the one in (8.2) under our choice (1.4). This parameter controls the splitting of massive $\mathcal{N}=$ 2 multiplets in the world-volume theory on the wall. It is not difficult to calculate this mass splitting, and so we did. If we substitute the expression for $\xi$ from Eq. (4.4) here, then (8.3) reduces to $\Delta m / m$. Note, however, that we can ignore the relation (4.4) coming from underlying non-Abelian theory and consider the $U(1)$ theory (4.2) on its own right. Then the $\mathcal{N}=2$ limit is characterized by parameters $\xi$ and $m^{A}$ while $\mu$ controls $\mathcal{N}=2$ breaking down to $\mathcal{N}=1$. In this setup the parameter of $\mathcal{N}=2$ breaking on the wall is given by (8.3) and goes to zero at $\mu \rightarrow 0$ while $\xi$ is fixed.

It is easy to see that with $f(a) \neq$ const the Bogomolny completion for the domain wall configuration is still possible, and we end up with the following first order equations:

$$
\begin{aligned}
\nabla_{z} \varphi^{A} & =-\frac{1}{\sqrt{2}} \varphi^{A}\left(a+\sqrt{2} m_{A}\right), \\
\partial_{z} a & =-\frac{g^{2}}{2 \sqrt{2}}\left(\left|\varphi^{A}\right|^{2}-f(a)\right),
\end{aligned}
$$

where the same ansatz as in Eq. (5.1) is used. These equations are quite similar to the equations (5.6) for $\mathcal{N}=2$ theory, the only difference being that the constant FI parameter $\xi$ is now replaced by a linear (and slowly-varying) function $f(a)$. The tension of this domain wall is still given by Eq. (5.8).

The emergence of the first order equations means that the wall at hand is still BPS saturated (albeit in $\mathcal{N}=1$ theory). This can be most straightforwardly seen by noting that a half of the relations (5.12) survive breaking of $\mathcal{N}=2$ to $\mathcal{N}=1$. Now we have four parameters $\varepsilon^{\alpha 1}$ and $\bar{\varepsilon}_{1 \dot{\alpha}}$, subject to two constrains,

$$
\bar{\varepsilon}_{1 \dot{2}}=-i \varepsilon^{21}, \quad \bar{\varepsilon}_{1 \mathrm{i}}=i \varepsilon^{11} .
$$

This leaves us with two supercharges which act trivially on the wall solution.

At small $\mu$ and $\Delta m \ll m$ the solution to first order equations (8.4) is a small perturbation of the domain wall solution presented in Sect. 5.2. Thus, it is still parametrized by two collective coordinates (moduli), $z_{0}$ and $\sigma$. In the exact $\mathcal{N}=$ 2 limit the moduli fields $\zeta(t, x, y)$ and $\sigma(t, x, y)$ formed the bosonic part of $\mathcal{N}=$ 2 vector multiplet in $2+1$ dimension. Now the question is: do these fields split once we break $\mathcal{N}=2$ supersymmetry softly down to $\mathcal{N}=1$ ? 
Naively one might think that while the "translational" field $\zeta(t, x, y)$ certainly remains massless, the second modulus field $\sigma(t, x, y)$ becomes massive acquiring a small mass, so that the $\mathcal{N}=2$ supermultiplet is split in two $\mathcal{N}=1$ supermultiplets. A particular mechanism for such splitting was suggested in Ref. [12] for the case of the domain wall interpolating between the monopole and dyon vacua. Namely, it was suggested that a Chern-Simons term is generated in the $(2+1)$-dimensional gauge theory on the wall, generating a mass to the $\mathrm{U}(1)$ gauge field. Although this conjecture is very relevant and will be exploited in Sec. 9.3, we will prove momentarily that the splitting does not take place for the wall interpolating between the quark vacua - the object of our study in this paper. The field $\sigma$ stays massless. In other words, the moduli field theory on the wall exhibits a supersymmetry enhancement - the particle contents is characteristic of the theory with four supercharges, rather than two supercharges one might expect to operate on the world sheet of $1 / 2$ BPS domain wall in $\mathcal{N}=1$ theory.

First, this conclusion follows from symmetry arguments. Indeed, the phase $\sigma$ is associated with the global $\mathrm{U}(1)$ rotation which is a part of the flavor $\mathrm{SU}\left(N_{f}=2\right)$ broken down to $\mathrm{U}(1)$ by the mass difference, $\Delta m \neq 0$. This symmetry is a vector-like and therefore is not anomalous. Thus, we have an exact global symmetry broken down on the domain wall solution. This implies the inevitability of the zero mode associated with the collective coordinate $\sigma$. With two massless moduli, $\zeta(t, x, y)$ and $\sigma(t, x, y), \mathcal{N}=1$ supersymmetry on the world sheet would imply two massless Majorana fields in the fermion sector. The fermion moduli are related to the fermion zero modes. It is instructive to check, by counting the fermion zero modes, that in the fermion sector we deal with two massless Majorana fields localized on the domain wall. We will now show that we have four fermion zero modes on the wall solution (two plus two complex conjugated).

To this end we use the Jackiw-Rebbi theorem which tells us that we have exactly two normalizable fermion zero modes (one two-component real fermion field) per each eigenvalue of the fermion mass matrix which changes its sign on the wall solution [22]. The fermion mass matrix in softly broken SQED is given by

$$
\begin{aligned}
S_{\text {mass }}^{\mathrm{ferm}} & =\frac{1}{\sqrt{2}} \int d^{4} x\left\{\bar{q}_{A f}\left(\lambda^{f} \psi^{A}\right)-\left(\tilde{\psi}_{A} \lambda_{f}\right) q^{f A}\right. \\
& \left.-\left(a+\sqrt{2} m_{A}\right)\left(\tilde{\psi}_{A} \psi^{A}\right)-\frac{\mu}{\sqrt{2}}\left(\lambda^{2}\right)^{2}\right\} .
\end{aligned}
$$

The last term explicitly breaks $\mathcal{N}=2$ supersymmetry and $S U(2)_{R}$ global symmetry because it depends only on the $f=2$ components of the field $\lambda^{f}$.

The fermion mass terms and Yukawa couplings in (8.6) give us a $6 \times 6$ fermion mass matrix. To study its eigenvalues we calculate its determinant. The general expression for the determinant is rather complicated; therefore, we study it approximately in different domains of the wall profile, see Fig. 3. The six eigenvalues of the mass matrix can be naturally divided in two classes: four "large" eigenvalues 
and two "small" ones.

\subsection{Fermion modes, $\mathcal{N}=2$ limit}

Consider first the $\mathcal{N}=2$ limit when the last term in Eq. (8.6) is ignored. Two "small" eigenvalues approach the photon mass (4.10) in both quark vacua,

$$
\left|\rho_{1,2}^{s}\right| \rightarrow m_{\gamma}, \quad z \rightarrow \pm \infty .
$$

The "large" eigenvalues behave as follows. In the left vacuum two of them approach the photon mass while the other two approach the larger value, $\Delta m$,

$$
\left|\rho_{1,2}^{l}\right| \rightarrow m_{\gamma}, \quad\left|\rho_{3,4}^{l}\right| \rightarrow \Delta m, \quad z \rightarrow-\infty
$$

while in the right vacuum their role is interchanged, namely

$$
\left|\rho_{3,4}^{l}\right| \rightarrow m_{\gamma}, \quad\left|\rho_{1,2}^{l}\right| \rightarrow \Delta m, \quad z \rightarrow \infty .
$$

This behavior is, of course, in perfect agreement with the mass spectrum of the theory in both quark vacua found in Sect. 4.2.

In the middle region $M$ (see Fig. 3) "large" eigenvalues interpolate between these two values. They always remain large inside the wall and clearly do not cross the zero. "Small" eigenvalues are exponentially small inside the wall and, therefore, one needs to carry out a more careful study. In the middle domain $M$ these eigenvalues are given by

$$
\rho_{1,2}^{s}= \pm \frac{1}{2}\left[\frac{g^{2}\left|\varphi^{1}\right|^{2}}{m_{1}+a / \sqrt{2}}+\frac{g^{2}\left|\varphi^{2}\right|^{2}}{m_{2}+a / \sqrt{2}}\right],
$$

where the functions $a$ and $\varphi^{A}$ are given by the domain wall solution (5.13), (5.17), and (5.19). Two "small" eigenvalues (8.10) clearly cross the zero at $z=z_{0}$, right in the middle of the domain wall. This is in accordance with our previous conclusion that we have four fermion zero modes in $\mathcal{N}=2$ limit.

\subsection{Fermion modes, $\mathcal{N}=2$ broken down to $\mathcal{N}=1$}

Now let us take into account the $\mathcal{N}=2$ breaking term in Eq. (8.6). Then the first eigenvalue in (8.10) gets an additional contribution

$$
\rho_{1}^{s}=\frac{1}{2}\left[\frac{g^{2}\left|\varphi^{1}\right|^{2}}{m_{1}+a / \sqrt{2}}+\frac{g^{2}\left|\varphi^{2}\right|^{2}}{m_{2}+a / \sqrt{2}}+2 g^{2} \mu\right],
$$

while the second one does not change. At small $\mu, \mu \ll \sqrt{\xi} / g$ both eigenvalues still cross the zero at $z=z_{0}$.

We conclude that in $\mathcal{N}=1$ theory the critical domain wall supports four fermion zero modes (at least in some domain of small $\mu$ and large $m$ ). This means that the 
$\mathcal{N}=2$ vector multiplet living on the wall ( 2 real boson fields +2 Majorana fields) is not split even after we break $\mathcal{N}=2$ supersymmetry down to $\mathcal{N}=1$. The lowenergy theory is still given by (5.39). Presumably, we would feel $\mathcal{N}=2$ breaking if we considered higher derivative terms on the world volume. We definitely see $\mathcal{N}=2$ breaking in the spectrum of massive excitations localized on the wall, with masses of the order of the inverse width of the wall $\sim 1 / R$.

\section{Comments on the literature}

The topic under consideration is rather hot; its aspects have been discussed in the recent literature in various contexts, including rather exotic, e.g. gauge field localization on branes in the framework of noncommutative field theories [36]. Here we briefly comment on the relation between our results and those one can fine in the literature.

\subsection{Generalities}

The most recent revival of the theme of field-theoretic implementation of D branes and strings can be attributed to Ref. [11] which presents an excellent elaboration of general ideas as to how gauge fields can be localized on domain walls. A variety of examples are worked out providing a clear-cut illustration to the statement [2] that localization of the gauge fields requires confinement in the bulk. It is also explained how this automatically entails the existence of the flux tubes ending on the walls. In Sec. 3 of Ref. [11] the authors construct a model for a (quasistable) wall-antiwall configuration of a variable thickness which traps a gauge field in the middle domain. This model served as an impetus for our construction, which, being totally different in many aspects, shares a common feature with that of Ref. [11] - the thickness of the middle domain in our model is a large variable parameter too.

\section{$9.2 \quad$ Varying $\Delta m$}

The proof of the existence of the "second" modulus $\sigma(t, x, y)$, dual to the $\mathrm{U}(1)$ gauge field on the wall, and its massless fermion superpartner was based on symmetry arguments and was independent on the value of the ratio $\Delta m / \sqrt{\xi}$. In our model this ratio is large. However, the $\mathrm{U}(1)$ gauge field localization must occur for arbitrary $\Delta m / \sqrt{\xi}$. This observation perfectly matches the result of Ref. [7], where a $1 / 4$ BPS solution of the string-wall junction type was found in a sigma model. In our language the sigma-model limit corresponds to $\Delta m / \sqrt{\xi} \rightarrow 0$. In this limit the photon field and its superpartners are heavy; upon integrating them out we recover the Kähler sigma model with the Eguchi-Hanson target space for the remaining light matter fields. It is well-known that such model have string-type solitons, which can have arbitrary transverse dimension (see Ref. [37] for a review of the so-called semilocal strings). 
Turning on $\Delta m \neq 0$ as a small perturbation produces a small potential on the target space. Once the potential is switched on, domain walls become possible, and one can search for the string-wall junctions. Strictly speaking the solution found in Ref. [7] is somewhat singular, since finite-radius strings exist only in the limit of massless sigma models while in this limit there are no domain walls. In massive sigma models treated in Ref. [7] the strings are forced to have vanishing transverse size, and, in fact, a "spike"-type junction was obtained. This is as close as one can get to $\mathcal{N}=2 \mathrm{SQCD}$ string-wall junctions in sigma models.

\subsection{Multiplicity of the Kaplunovsky-Sonnenschein-Yankielo- wicz domain walls}

In this section we leave the solid ground of weakly coupled models and venture into uncharted waters of strong coupling. The issue to be addresses is the domain wall connecting the monopole and dyon vacua in $\mathcal{N}=2$ SQCD slightly perturbed by $\mu \operatorname{Tr} \Phi^{2}$ term (with $\mathrm{SU}(2)$ gauge group). This problem has been recently studied [23] by Kaplunovsky et al. (KSY for brevity). We would like to address the issue of multiplicity of such domain walls (i.e. the number of distinct domain walls interpolating between the given vacua, with degenerate tensions).

First of all, let us summarize what is known about the multiplicity of the superYang-Mills walls at strong coupling. This question was analyzed by Acharya and Vafa, Ref. [12], from the string theory side. Representing the (1+2)-dimensional domain wall of the super-Yang-Mills theory as a D4-brane wrapped over $S^{2}$, the authors found that the $\mathrm{U}(1)$ gauge field localized on the wall is described by supersymmetric QED (similar to the construction discussed in Sec. 5.4) with the Chern-Simons term at level $N$,

$$
\mathcal{L}_{1+2}=-\frac{1}{4 e_{\text {eff }}^{2}} F_{m n} F^{m n}+\frac{N}{16 \pi} F_{m n} A_{k} \varepsilon^{m n k}+\text { ferm. terms }
$$

where $N$ is related to $\mathrm{SU}(N)$ of the underlying (1+3)-dimensional gauge theory. For the $\mathrm{SU}(2)$ gauge group $N=2$. It is well-known that the level of the Chern-Simons term determines the number of vacua of the theory - two in the case at hand (the gauge group of the underlying theory is $\mathrm{SU}(2)$ ). The number of vacua in the $(1+2)$-dimensional effective theory on the wall is the number of distinct degenerate domain walls in $(1+3)$-dimensional theory (the above "two" refers to the counting convention explained in Sect. 5.6).

Needless to say, this is an index of the underlying theory, which does not change under continuous deformations of the theory. Basing on this fact, the domain-wall multiplicity was calculated directly from field theory [13], and was found to coincide with the Acharya-Vafa result.

Let us return now to Kaplunovsky et al. Since they considered $\mathcal{N}=2 \mathrm{SQCD}$ perturbed by $\mu \operatorname{Tr} \Phi^{2}$ term, and at large $\mu$ this theory smoothly goes into $\mathcal{N}=$ 
1 gluodynamics, the index argument tells us that the number of distinct KSY walls, in the case of the $\mathrm{SU}(2)$ gauge group, is two.

Near each vacuum - monopole and dyon - Kaplunovsky et al. use distinct effective low-energy SQED-type descriptions, e.g. near the monopole vacuum the lowenergy model includes a U(1) gauge field and its superpartners, plus light monopole superfield $\mathcal{M}, \tilde{\mathcal{M}}$. Near the dyon vacuum it is also a $\mathrm{U}(1)$ gauge superfield (albeit not that of the previous patch) plus a light dyon superfield $\mathcal{D}, \tilde{\mathcal{D}}$. In the intermediate patch the authors keep just one superfield — that of $u=\operatorname{Tr} \Phi^{2}$. It is worth stressing that no unified description exists and the consideration has to be carried out in three distinct patches separately. A unique solution to the Bogomolny equations was found.

The KSY solution bears a remarkable resemblance to the domain wall in our weakly coupled model. Indeed, the KSY wall consists of three domains - a broad middle domain of size $\sim \mu^{-1}$, where the monopole and dyon condensates do vanish, and two much narrower edge domains (of thickness $\sim(\mu \Lambda)^{-1 / 2}$ ) where the transition from the vacuum value of the condensate $\mathcal{M} \tilde{\mathcal{M}}$ (or $\mathcal{D} \mathcal{D}$ ) to zero occurs. So, why we speak of an unsolved problem?

That's because the KSY solution shows no sign of two distinct domain walls. As was explained above, the double degeneracy of the domain wall is a must. Since the index does not depend on the value of $\mu$, the limit of small $\mu$ considered in Ref. [23] must exhibit the same number of domain walls as the one emerging in the large $\mu$ limit.

What is lacking in Ref. [23]? An obvious analogy with our weakly coupled model prompts us that the lacking element is the analysis of moduli (or quasimoduli) fields localized on the wall.

For the domain wall at weak coupling, considered in the present paper, there is an unambiguous supersymmetry-based argument proving the double degeneracy. In the $\mathcal{N}=2$ limit our BPS wall belongs to the short representation of $\mathcal{N}=2$ superalgebra, i.e. we have two boson + two fermion states (4 fermion zero modes). When we break $\mathcal{N}=2$ down to $\mathcal{N}=1$ our wall is still $\mathcal{N}=1$ BPS - it belongs to a short representation of $\mathcal{N}=1$ superalgebra. This is possible only if we have two $\mathcal{N}=1$ short multiplets because the number of states cannot discontinuously change. One of these supermultiplets is the translational modulus plus its superpartner, another is the phase field $\sigma$ and its superpartner.

For the KSY wall, generally speaking, this argument does not apply because we do not have $\mathcal{N}=2$ limit: the $\mathcal{N}=2$ breaking parameter (8.3) is never small. Why, nevertheless, we suggest that the missed multiplicity of the KSY walls might be associated with a missed (quasi)modulus?

In the KSY problem, there are two distinct dynamically generated phase symmetries - one associated with the phase rotations of $\mathcal{M}$, another with the phase rotations of $\mathcal{D}$. At the same time the $\mathrm{U}(1)$ gauge field is single (though it is described differently in the two edge patches). There are no massless particles in either of the vacua. So far, all this is perfectly parallel to what we have in our model. 
Now the two theories divorce. Ours has an exact global U(1), unbroken in the vacua and spontaneously broken on the wall, which results in the strictly massless modulus $\sigma(t, x, y)$. The Seiberg-Witten theory perturbed by $\mu \operatorname{Tr} \Phi^{2}$ (the KSY case) has no strictly conserved $\mathrm{U}(1)$. Due to the full similarity in the description of the edge domains, one may expect, however, the emergence of a quasimodulus localized on the KSY wall. We will call it $\tilde{\sigma}(t, x, y)$.

To make quasimodulus $\tilde{\sigma}$ massive we have to include a periodic superpotential, of the type $\cos (\tilde{\sigma})$ giving rise to a scalar potential of the type $\sin ^{2}(\tilde{\sigma})$ which has two vacua. This is quite a general argument based only on periodicity in $\tilde{\sigma}$ and on the existence of the quasimodulus field localized on the KSY domain wall, our basic conjecture in this Section.

The quasimodulus field with the target space on $S_{1}$, (with a non-vanishing mass) can be transformed into a dual $(2+1)$-dimensional QED with a Chern-Simons term. In this way we match with the Acharya-Vafa analysis.

A slightly different argument for this quasimodulus field $\tilde{\sigma}(t, x, y)$ is as follows. In each of the edge domains of the wall (i.e. near the monopole and dyon vacua) the underlying theory is approximately $\mathcal{N}=2$. The parameter governing the breaking of $\mathcal{N}=2$ is of the order $\sqrt{\mu / \Lambda}$. In the middle of the wall, the breaking of $\mathcal{N}=2$ is stronger, but it is natural to think that the phase field $\tilde{\sigma}$ is essentially disassociated from the middle domain. Then the effective theory on the wall must be close to $(2+1)$-dimensional $\mathcal{N}=2$, which would require two real massless bosonic moduli. The breaking of $\mathcal{N}=2$ splits the supermultiplet into two $(2+1)$-dimensional $\mathcal{N}=1$ supermultiplets as now there is no exact $\mathrm{U}(1)$ to prevent the splitting. A natural estimate for the mass of the quasimodulus $\tilde{\sigma}$ is then

$$
m_{\tilde{\sigma}} \sim \sqrt{\mu \Lambda} \sqrt{\mu / \Lambda} \sim \mu .
$$

The dual $(2+1)$-dimensional SQED will have the Lagrangian (9.1) with $e_{\mathrm{eff}}^{2}=\mu / \kappa$ where $\kappa$ is a dimensionless constant of order one. The value of the constant in front of $F_{m n} F^{m n}$ will be in accord with our result (5.40), since the thickness of the KSY wall is $\sim \mu^{-1}$.

\section{Brief conclusions}

We suggest and work out a model which seems to be a good prototype for studying basic properties of D brane/string theory in the field-theoretic setting. Our model is weakly coupled, fully controllable theoretically and possesses both critical walls and strings.

Then, using our model as a tool we addressed in a fully quantitative manner the following long-standing issues:

(i) gauge field localization on the wall;

(ii) the wall-string junction (i.e. a flux tube coming from infinity and ending on the all). 
We confirm that $1 / 2$ BPS domain wall does localize a U(1) gauge field; the charge which presents the source for this field is confined in the bulk.

We find that an $1 / 2$ BPS flux tube coming from infinity does indeed end on the above wall. The wall-string junction is $1 / 4$ BPS.

A task which remains for the future is (the quantitative analysis of) localization of non-Abelian gauge fields on the wall and related flux-tube-wall junctions.

\section{Acknowledgments}

We are grateful to Alexander Gorsky, Adam Ritz and Arkady Vainshtein for helpful discussions. The work of M. S. is supported in part by DOE grant DE-FG0294ER40823, A. Y. is supported in part by the Russian Foundation for Basic Research grant No. 02-02-17115, by INTAS grant No. 00-00334 and by Theoretical Physics Institute at the University of Minnesota.

\section{References}

[1] J. Polchinski, Phys. Rev. Lett. 75, 4724 (1995) [hep-th/9510017]; see also J. Polchinski, String Theory, (Cambridge University Press, 1998), Vols. 1 and 2.

[2] G. R. Dvali and M. A. Shifman, Phys. Lett. B 396, 64 (1997) (E) B 407, 452 (1997) [hep-th/9612128].

[3] E. Witten, Nucl. Phys. B 507, 658 (1997) [hep-th/9706109].

[4] I. I. Kogan, A. Kovner and M. A. Shifman, Phys. Rev. D 57, 5195 (1998) [hep-th/9712046].

[5] A. Hanany, M. Strassler and A. Zaffaroni, Nucl. Phys. B513, 87 (1998) [hepth/9707244].

[6] A. Vainshtein and A. Yung, Nucl. Phys. B614, 3 (2001) [hep-th/0012250].

[7] J. P. Gauntlett, R. Portugues, D. Tong and P. K. Townsend, Phys. Rev. D 63, 085002 (2001) [hep-th/0008221].

[8] N. Seiberg and E. Witten, Nucl. Phys. B426, 19 (1994) [hep-th/9407087].

[9] N. Seiberg and E. Witten, Nucl. Phys. B431, 484 (1994) [hep-th/9408099].

[10] S. L. Dubovsky and V. A. Rubakov, Int. J. Mod. Phys. A 16, 4331 (2001) [hep-th/0105243].

[11] G. Dvali and A. Vilenkin, hep-th/0209217. 
[12] B. S. Acharya and C. Vafa, hep-th/0103011.

[13] A. Ritz, M. Shifman and A. Vainshtein, Phys. Rev. D 66, 065015 (2002) [hepth/0205083].

[14] X. Hou, Phys. Rev. D 63, 045015 (2001) [hep-th/0005119].

[15] P. Fayet and J. Iliopoulos, Phys. Lett. B 51, 461 (1974).

[16] A. Yung, Nucl. Phys. B 562, 191 (1999) [hep-th/9906243]; Nucl. Phys. B 626, 207 (2002) [hep-th/0103222];

A. Marshakov and A. Yung, Nucl. Phys. B 647, 3 (2002) [hep-th/0202172].

[17] A. Abrikosov, Sov. Phys. JETP 321442 (1957) [Reprinted in Solitons and Particles, Eds. C. Rebbi and G. Soliani (World Scientific, Singapore, 1984), p. 356];

H. Nielsen and P. Olesen, Nucl. Phys. B61 45 (1973) [Reprinted in Solitons and Particles, Eds. C. Rebbi and G. Soliani (World Scientific, Singapore, 1984), p. 365].

[18] W. Garcia Fuertes and J. Mateos Guilarte, Phys. Lett. B 437, 82 (1998) [hepth/9807218].

[19] J. D. Edelstein, W. G. Fuertes, J. Mas and J. M. Guilarte, Phys. Rev. D 62 , 065008 (2000) [hep-th/0001184].

[20] K. Konishi and L. Spanu, hep-th/0106175.

[21] A. M. Polyakov, Nucl. Phys. B 120, 429 (1977).

[22] R. Jackiw and C. Rebbi, Phys. Rev. D 13, 3398 (1976) [Reprinted in Solitons and Particles, Eds. C. Rebbi and G. Soliani, (World Scientific, Singapore, 1984), pp. 331-342].

[23] V. S. Kaplunovsky, J. Sonnenschein and S. Yankielowicz, Nucl. Phys. B 552, 209 (1999) [hep-th/9811195].

[24] A. Kovner, M. A. Shifman and A. Smilga, Phys. Rev. D 56, 7978 (1997) [hepth/9706089].

[25] J. P. Gauntlett, D. Tong and P. K. Townsend, Phys. Rev. D 64, 025010 (2001) [hep-th/0012178].

[26] E. B. Bogomolny, Yad. Fiz. 24, 861 (1976) [Sov. J. Nucl. Phys. 24, 449 (1976), reprinted in Solitons and Particles, Eds. C. Rebbi and G. Soliani (World Scientific, Singapore, 1984), p. 389].

[27] E. Witten and D. I. Olive, Phys. Lett. B 78, 97 (1978). 
[28] J. A. de Azcarraga, J. P. Gauntlett, J. M. Izquierdo and P. K. Townsend, Phys. Rev. Lett. 63, 2443 (1989).

[29] Z. Hlousek and D. Spector, Nucl. Phys. B 370, 143 (1992);

J. Edelstein, C. Nunez and F. Schaposnik, Phys. Lett. B 329, 39 (1994) [hepth/9311055].

[30] S. C. Davis, A. C. Davis and M. Trodden, Phys. Lett. B 405, 257 (1997) [hepph/9702360].

[31] A. Gorsky and M. A. Shifman, Phys. Rev. D 61, 085001 (2000) [hepth/9909015].

[32] M. Shifman and A. Yung, Phys. Rev. D 66, 045012 (2002) [hep-th/0205025].

[33] D. Tong, Phys. Rev. D 66, 025013 (2002) [hep-th/0202012].

[34] K. S. Lee, hep-th/0211058.

[35] E. Witten, Nucl. Phys. B 500, 3 (1997) [hep-th/9703166].

[36] S. L. Dubovsky, V. A. Rubakov and S. M. Sibiryakov, JHEP 0201, 037 (2002) [hep-th/0201025].

[37] A. Achucarro and T. Vachaspati, Phys. Rept. 327, 347 (2000) [Phys. Rept. 327, 427 (2000)] [hep-ph/9904229]. 